\title{
Involvement of Arabidopsis Multi-Copper Oxidase-Encoding LACCASE12 in Root-to-Shoot Iron Partitioning: A Novel Example of Copper-Iron Crosstalk
}

\author{
María Bernal1,2* and Ute Krämer ${ }^{1 *}$ \\ ${ }^{1}$ Department of Molecular Genetics and Physiology of Plants, Faculty of Biology and Biotechnology, Ruhr University \\ Bochum, Bochum, Germany, ${ }^{2}$ Department of Plant Nutrition, Estación Experimental de Aula Dei-CSIC, Zaragoza, Spain
}

OPEN ACCESS

Edited by:

Francisco Javier Romera, University of Córdoba, Spain

Reviewed by: Ricardo Fabiano Hettwer Giehl, Leibniz Institute of Plant Genetics and Crop Plant Research (IPK),

Germany

Diego F. Gomez-Casati,

National University of Rosario,

Argentina

Olena Vatamaniuk,

Cornell University, United States

*Correspondence:

María Bernal

maria.bernal@ruhr-uni-bochum.de

Ute Krämer

ute.kraemer@ruhr-uni-bochum.de

Specialty section:

This article was submitted to

Plant Nutrition,

a section of the journal

Frontiers in Plant Science

Received: 30 March 2021

Accepted: 23 August 2021

Published: 11 October 2021

Citation:

Bernal M and Krämer U (2021)

Involvement of Arabidopsis

Multi-Copper Oxidase-Encoding

LACCASE12 in Root-to-Shoot Iron

Partitioning: A Novel Example

of Copper-Iron Crosstalk.

Front. Plant Sci. 12:688318.

doi: 10.3389/fp/s.2021.688318
Numerous central biological processes depend on the participation of the essential elements iron $(\mathrm{Fe})$ or copper $(\mathrm{Cu})$, including photosynthesis, respiration, cell wall remodeling and oxidative stress protection. Yet, both $\mathrm{Fe}$ and $\mathrm{Cu}$ metal cations can become toxic when accumulated in excess. Because of the potent ligand-binding and redox chemistries of these metals, there is a need for the tight and combined homeostatic control of their uptake and distribution. Several known examples pinpoint an inter-dependence of $\mathrm{Fe}$ and $\mathrm{Cu}$ homeostasis in eukaryotes, mostly in green algae, yeast and mammals, but this is less well understood in multicellular plants to date. In Arabidopsis, Cu deficiency causes secondary Fe deficiency, and this is associated with reduced in vitro ferroxidase activity and decreased root-to-shoot Fe translocation. Here we summarize the current knowledge of the cross-talk between $\mathrm{Cu}$ and Fe homeostasis and present a partial characterization of LACCASE12 (LAC12) that encodes a member of the multicopper oxidase (MCO) protein family in Arabidopsis. LAC12 transcript levels increase under Fe deficiency. The phenotypic characterization of two mutants carrying T-DNA insertions suggests a role of LAC12 in root-to-shoot Fe partitioning and in maintaining growth on Fe-deficient substrates. A molecular understanding of the complex interactions between $\mathrm{Fe}$ and $\mathrm{Cu}$ will be important for combating Fe deficiency in crops and for advancing biofortification approaches.

Keywords: copper, iron, multicopper oxidase, homeostasis, deficiency

\section{INTRODUCTION}

Copper $(\mathrm{Cu})$ and iron $(\mathrm{Fe})$ are essential micronutrients for plants and most other forms of life. Both elements function as cofactors of important enzymes in a large number of biochemical pathways due to their redox chemical properties, as for example the electron transport chains involved in photosynthesis and mitochondrial respiration (Nouet et al., 2011; Ravet and Pilon, 2013). Fe also plays an important role in chlorophyll biosynthesis, sulfate assimilation, hormone signaling, nitrogen metabolism and DNA synthesis and repair. $\mathrm{Cu}$ is also required in ethylene perception, cell wall metabolism and oxidative stress responses (Printz et al., 2016). The importance of both micronutrients is even more evident through the deleterious effects that their corresponding deficiencies provoke in plants. Common symptoms of $\mathrm{Cu}$ deficiency are plant chlorosis in young tissues and necrosis in reproductive organs (Tewari et al., 2006; Printz et al., 2016). Fe deficiency 
causes severe chlorosis, and is a major restriction for crop yield and nutritional quality (Marschner, 1995; Puig et al., 2007; Connorton et al., 2017; Kroh and Pilon, 2020). The same redox properties of $\mathrm{Cu}$ and $\mathrm{Fe}$ that are crucial for the correct function of a wide range of proteins, can become toxic when these metals are accumulated in excess. Both excess $\mathrm{Fe}$ and excess $\mathrm{Cu}$ can induce the uncontrolled production of reactive oxygen species (ROS), combined with between-metal interference and competition (Ravet and Pilon, 2013). Consequently, there is a need for a tight homeostatic control of uptake and distribution of both metals.

The impact of plant nutrition on human health is large. The World Health Organization (WHO) estimates that 30\% of the world's population $^{1}$ are not taking in adequate amounts of $\mathrm{Fe}$ and therefore suffering from Fe-deficiency anemia. In infancy, for example, insufficient $\mathrm{Fe}$ intake can result in cognitive limitations that persist into adulthood (Collins et al., 2010). Additionally, it is well known that $\mathrm{Fe}$ and $\mathrm{Cu}$ also interact in the human diet. Both $\mathrm{Fe}$ and $\mathrm{Cu}$ deficiency can co-occur in selected populations consuming normal diets (Collins et al., 2010). To handle better these micronutrient deficiencies, a number of different strategies are being developed, as for instance oral additives, enriching prepared foods by adding supplements, and spraying diverse crop plants with fertilizers containing $\mathrm{Cu}$ or Fe (Zhao and McGrath, 2009; Shahzad et al., 2014). Another approach is to screen plants for identifying varieties with enhanced levels of $\mathrm{Fe}$ or $\mathrm{Cu}$ in their grains or in other edible tissues, followed if necessary, by using certain genetic markers to later breed only with chosen varieties. This approach is known as biofortification. Targeted biofortification approaches are also promising, as exemplified in the study in which increased production of ferritins or nicotianamine synthases could help to increase the Fe content in rice grains (Masuda et al., 2013). However, the accumulation of excess concentrations of the same essential metals can also cause human diseases as for example Wilson's disease $(\mathrm{Cu})$ or hemochromatosis (Fe) (Dusek et al., 2015). Thus, these metals are a risk for human health. Additionally, overaccumulation of these metals can contaminate plants, livestock and wildlife (Nriagu and Pacyna, 1988). The position of plants at the beginning of the food chain implies that an improved understanding of metal homeostasis of higher plants is fundamentally important for the environment, agriculture and human health.

Higher plants have established systemic homeostatic processes to efficiently take up, distribute and use micronutrients such as $\mathrm{Cu}$ and $\mathrm{Fe}$. The molecular mechanisms of $\mathrm{Cu}$ and Fe homeostasis are quite well understood individually. However, an understanding of the cross-talk among the homeostasis of these metals is only beginning to arise (Table 1). Metalloprotein substitution is one well-characterized example of cross-talk between $\mathrm{Cu}$ and $\mathrm{Fe}$ homeostasis in higher plants (Yamasaki et al., 2007; Waters et al., 2012; Pilon, 2017). In Arabidopsis thaliana, when $\mathrm{Cu}$ is scarce, CU/ZN SUPEROXIDE DISMUTASES 1 and 2 (CSD1 and CSD2) are replaced by FE SUPEROXIDE DISMUTASE 1 (FSD1), most likely to reorganize metabolism to economize on $\mathrm{Cu}$ for maintaining the functions of essential proteins

${ }^{1}$ http://www.who.int/nutrition/topics/ida/en/ such as PLASTOCYANIN (PC). A metalloprotein substitution mechanism of economizing on $\mathrm{Cu}$ was first identified in the green alga Chlamydomonas reinhardtii. When this green alga grows under $\mathrm{Cu}$ deficiency conditions, plastocyanin is replaced by the haem-containing cytochrome $c_{6}$ (Quinn and Merchant, 1995; Kropat et al., 2005). This substitution mechanism is regulated by the transcription factor COPPER RESPONSE REGULATOR (CRR1) in Chlamydomonas. In Arabidopsis, the homologous SQUAMOSA PROMOTER BINDING PROTEIN-LIKE7 (SPL7) (Yamasaki et al., 2009; Bernal et al., 2012) operates through microRNA 398 (miR398) (Yamasaki et al., 2007). Under Cu deficiency, SPL7 binds to GTAC motifs in the promoter regions of target genes and activates the transcription of FSD1 as well as of MIR398, among other Cu-miRNAs, which in turn suppress CSD1 and CSD2 transcript levels. miR398 also regulates COPPER CHAPERONE FOR SOD (CCS) transcript levels (Sunkar and Zhu, 2004; Yamasaki et al., 2009; Beauclair et al., 2010). Additionally, under Fe deficiency, FSD1 is substituted by CSD1 and CSD2. This operates via the downregulation of miR398 under Fe deficiency, which causes the upregulation of CSD1 and CSD2 transcripts (Waters et al., 2012). Another so-called $\mathrm{Cu}$-microRNA, miR408, is also described to participate in the metabolic adjustment to low $\mathrm{Cu}$ supply (Buhtz et al., 2010; Carrió-Seguí et al., 2019b). Under Cu deficiency conditions, the transcription of MIR408 is upregulated by SPL7 via the binding to the GTAC motifs within the MIR408 promoter (Yamasaki et al., 2009; Bernal et al., 2012; Zhang and Li, 2013). miR408 mediates the post-transcriptional downregulation of the LAC3, LAC12, and LAC13 mRNAs encoding LACCASE-LIKE MULTICOPPER OXIDASES (LMCOs) (Abdel-Ghany and Pilon, 2008). The $A$. thaliana genome contains 17 loci encoding laccaselike multicopper oxidases (LMCOs). In all LMCO proteins the characteristic $\mathrm{Cu}$ binding motifs associated with MCO functionality are conserved (Supplementary Figure 1) (Turlapati et al., 2011). Laccases are glycoproteins that catalyze the oxidation in vitro of a wide variety of aromatic substrates including phenolic compounds and amines (McCaig et al., 2005; Turlapati et al., 2011). Based on this, LMCOs could have more diverse functions than initially expected (Reiss et al., 2013). However, their precise physiological/biochemical roles in higher plants remain largely unclear. These proteins could be involved in various physiological processes such as $\mathrm{Cu}$ deficiency response, Fe metabolism, lignification, and oxidative stress (McCaig et al., 2005; Turlapati et al., 2011).

Arabidopsis plants with modified miR408 levels experience the deregulation of several processes under Fe-deficient conditions, as for instance a reduction of the chlorophyll and lignin content and deregulation of the expression of several oxidative stress and lignin biosynthesis genes (Carrió-Seguí et al., 2019b). This suggests a potential role of LMCOs in Fe deficiency. However, the signaling pathway that drives a repression of miR408 levels under Fe deficiency conditions and its physiological relevance remain unknown (Carrió-Seguí et al., 2019b). MIR408 was also identified as a target gene of ELONGATED HYPOCOTYL 5 (HY5) through the binding to G-box motifs. HY5 is a transcription factor that regulates light signaling and physically interacts with SPL7 (Zhang et al., 2014). 
HY5 and SPL7 can simultaneously bind to the MIR408 promoter and coordinately control miR408 levels and its target genes in response to light and $\mathrm{Cu}$, with SPL7 having a stronger impact on miR408 levels. The constitutive production of miR408 was reported to rescue the developmental phenotypes of the hy5, spl7 and hy 5 spl7 mutants under $\mathrm{Cu}$ deficiency conditions. These results suggested that miR408 mediates light-copper crosstalk (Zhang et al., 2014). In a variety of plant species such as tobacco and rice, the overexpression of MIR408 improves photosynthesis and growth rates as well as seed yield (Zhang et al., 2017; Pan et al., 2018; Song et al., 2018).

Another interaction between $\mathrm{Cu}$ and $\mathrm{Fe}$ homeostasis in A. thaliana involves the $\mathrm{Cu}$ TRANSPORTER (COPT) family, members of which generally mediate $\mathrm{Cu}(\mathrm{I})$ import into the cytosol (Sancenón et al., 2003). Among the COPT genes, COPT2 is a plasma membrane protein that functions in $\mathrm{Cu}$ uptake. Under $\mathrm{Cu}$ deficiency, COPT2 is expressed most highly in the roots and is upregulated in dependence on SPL7 (Yamasaki et al., 2009; Bernal et al., 2012). Additionally, COPT2 was shown to contribute to Fe homeostasis (Perea-García et al., 2013). COPT2 transcript levels are upregulated in response to Fe deficiency and this regulation partially depends on FER-LIKE IRON DEFICIENCY INDUCED TRANSCRIPTION FACTOR (FIT), one of the principal transcription factors that regulate the Fe deficiency response in Arabidopsis (Colangelo and Guerinot, 2004). A potential explanation for this regulation may be a prioritization of a $\mathrm{Cu}$ reallocation to $\mathrm{Cu}$-dependent enzymes such us CSD1 and CSD2, which replace FSD1 when Fe levels are low and minimize further Fe consumption (Waters et al., 2012; Perea-García et al., 2013). The dual regulation of COPT2 by FIT and SPL7 may explain the role of the encoded copper transporter in the interaction between the metal homeostasis of both metals. A simultaneous $\mathrm{Cu}$ and Fe deficiency study shows that copt2 mutant plants are more tolerant than the wild-type plants, with an alleviation of leaf chlorosis, an increase of chlorophyll concentration and higher plastocyanin concentrations, resulting in improved growth and seed yield (Perea-García et al., 2013). In addition, based on gene expression analysis, a link is suggested between COPT2-mediated $\mathrm{Cu}$ uptake through the $\mathrm{Cu}$ transporter COPT2 and phosphate $(\mathrm{Pi})$ starvation signaling, which is strongly related to Fe homeostasis (Ward et al., 2008; Perea-García et al., 2013). Transcript profiling of copt2 and wild-type plants grown under Fe deficiency and simultaneous $\mathrm{Fe}$ and $\mathrm{Cu}$ deficiency, respectively, show that a relevant number of the upregulated transcripts are associated to Pi starvation. Under Pi starvation, the copt2 phenotype is not affected, and the transcript levels of COPT2 are not changed in wild-type Arabidopsis plants. Taken together, these observations suggest that COPT2 function affects the regulation of $\mathrm{Cu}, \mathrm{Fe}$ and $\mathrm{Pi}$ deficiency responses (Perea-García et al., 2013).

The role of other members of the COPT family in both $\mathrm{Cu}$ and $\mathrm{Fe}$ homeostasis was also studied. To analyze the interconnection between internal vacuolar pools of $\mathrm{Fe}$ and $\mathrm{Cu}$, loss-of-function mutants of the tonoplast $\mathrm{Cu}$ transporter COPT5 and the Fe transporters NATURAL RESISTANCEASSOCIATED MACROPHAGE PROTEIN 3/4 (NRAMP3 and NRAMP4), respectively, were characterized (Carrió-Seguí et al., 2019a). This study demonstrates that the copt5 mutant is affected by $\mathrm{Fe}$ deficiency and the growth of the nramp3nramp4 double mutant is strongly damaged under $\mathrm{Cu}$ deficiency. copt5 mutant plants show a strong upregulation of NRAMP4 transcript levels whereas in the nramp3nramp4 mutant COPT5 transcripts are highly expressed under $\mathrm{Cu}$ deficiency. The authors suggested that the regulation of vacuolar $\mathrm{Cu}$ and Fe pools is interconnected, the absence of a functional tonoplast metal transporter for one of the metals provokes the reallocation of the other metal and the induction of its respective metal transporter (CarrióSeguí et al., 2019a). Additionally, the overexpression of the $\mathrm{Cu}$

TABLE 1 | Overview of the publications reporting cross-talk between Fe and Cu homeostasis in plants.

\section{Summary}

Cu transporter COPT2 transcript levels are upregulated by FIT under Fe-deficient conditions

Reciprocal regulation of SODs via miR398

Role of microRNAs in the regulation of non-essential cuproproteins

Fe deficiency responses include accumulation of $\mathrm{Cu}$, regulation of microRNAs, and differential expression of Fe and CuSOD genes

Cu deficiency induces Fe deficiency in Arabidopsis, and this is associated with lowered root-to-shoot translocation of Fe copt2 mutants are more tolerant to simultaneous Fe and Cu deficiency as well as more insensitive to phosphate deficiency LPR1 and LPR2, two MCO proteins, act as ferroxidases in Arabidopsis

COPT1 overexpressor rice plants show high Fe content in grains

SPL7-dependent repression of some components of the Fe uptake, and the ethylene response factor (ERF) redox homeostasis network

Arabidopsis plants with modified miR408 levels experience de-regulation of several processes under Fe-deficient conditions

The loss-of-function of COPT5 induces the remobilization of the vacuolar Fe pool by inducing the expression of the Fe vacuolar transporter NRAMP3 NRAMP4

A simultaneous $\mathrm{Cu}$ and Fe deficiency study in Arabidopsis shows a reconfiguration of large sets of molecules, such as central carbon metabolites, in particular photosynthates and amino acids

Arabidopsis plants overexpressing COPT1 or COPT3 are not able to properly respond to Fe deficiency revealing the importance of an appropriate spatiotemporal Cu uptake for Fe homeostasis

A reduction of the fumarate synthesis in the cytosol increases the Fe availability for metalloproteins in - Cu - Fe medium

\section{Publication}

Colangelo and Guerinot, 2004

Yamasaki et al., 2007

Abdel-Ghany and Pilon, 2008

Waters et al., 2012

Bernal et al., 2012

Perea-García et al., 2013

Müller et al., 2015

Andrés-Bordería et al., 2017

Kastoori Ramamurthy et al., 2018

Carrió-Seguí et al., 2019b

Carrió-Seguí et al., 2019a

Garcia-Molina et al., 2020

Perea-García et al., 2020 
transporter COPT1 in rice plants induces the accumulation of $\mathrm{Fe}$ in rice grains (Andrés-Bordería et al., 2017). Furthermore, Arabidopsis plants overexpressing COPT1 or COPT3 grown under $\mathrm{Cu}$ deficiency conditions cannot properly respond to $\mathrm{Fe}$ deficiency. These plants show an altered expression of several Fe homeostasis genes, including genes of the Fe uptake system and their transcriptional regulators. This suggests the relevance of a correct interconnection between $\mathrm{Cu}$ and $\mathrm{Fe}$ homeostasis under metal deficiency growth conditions (Perea-García et al., 2020).

A study addressing the role of SPL7 in Fe homeostasis of Arabidopsis hypothesized on new potential functions for SPL7 as a transcriptional repressor of several components of the Fe uptake machinery, such as the IRON MAN/FEP1 (IMA/FEP) peptides and the subgroup Ib of BASIC HELIXLOOP-HELIX $(b H L H)$ transcription factor, as well as of genes of the ethylene response factor (ERF) redox homeostasis network (Kastoori Ramamurthy et al., 2018). Simultaneous $\mathrm{Cu}$ and Fe deficiency $(-\mathrm{Cu}-\mathrm{Fe})$ in Arabidopsis leads a reconfiguration of a huge number of molecules, such as central carbon metabolites, including a decrease of photosynthates and an increase of free amino acids under $-\mathrm{Cu}-\mathrm{Fe}$ conditions. This was proposed to be associated with a swap from autotrophic to heterotrophic growth, and also includes organic acids like fumaric acid in the response to both deficiencies that may help plants to acclimate to simultaneous - $\mathrm{Cu}$-Fe deficiency (Garcia-Molina et al., 2020). A more recent publication reports that the availability of cytosolic $\mathrm{Fe}$ is increased when the synthesis of cytosolic fumarate is decreased (Garcia-Molina et al., 2021).

In yeast and humans, $\mathrm{Cu}$ is essential for the uptake and distribution of Fe (Muckenthaler et al., 2008). In these organisms, the activity of MULTICOPPER OXIDASES (MCOs), which contain $\mathrm{Cu}$ as a cofactor, of oxidizing $\mathrm{Fe}(\mathrm{II})$ to $\mathrm{Fe}$ (III) is indispensable for the operation of specific transmembrane $\mathrm{Fe}$ transport roles (Askwith et al., 1994; Harris et al., 1995; Muckenthaler et al., 2008; Gulec and Collins, 2014). In yeast, $\mathrm{Fe}$ uptake involves the prior extracellular reduction of ferric chelates by the cell surface reductases FRE1 and FRE2. Subsequently, $\mathrm{Fe}^{2+}$ cations are reoxidized to $\mathrm{Fe}^{3+}$ by the multicopper oxidase FET3, which acts as a ferroxidase, and $\mathrm{Fe}^{3+}$ cations are subsequently imported through the transmembrane permease FTR1. This FET3-FTR1 complex is known as the high affinity Fe uptake system. As a consequence, $\mathrm{Cu}$ deficiency interferes with high-affinity Fe uptake into yeast cells and can cause Fe deficiency symptoms (Askwith et al., 1994). In addition, the plasma membrane transporter FET4 mediates low-affinity $\mathrm{Fe}^{2+}$ uptake. Different from yeast, multicopper ferroxidases are required for cellular Fe export in humans. There are three key multicopper ferroxidases, caeruloplasmin, haephestin and zyklopen, which act in conjunction with the plasma membrane $\mathrm{Fe}^{2+}$ exporter ferroportin 1. Caeruloplasmin acts in physiological processes, including $\mathrm{Cu}$ transport and biogenic amine oxidation, and it is an essential ferroxidase in human Fe homeostasis. Haephestin is responsible for allowing the transport of dietary Fe from intestinal enterocytes into the bloodstream. Zyklopen functions to oxidize the ferrous iron to ferric iron after transfer through ferroportin from placental trophoblasts from mother to fetus (Hellman and
Gitlin, 2002; Chen et al., 2010; Gulec and Collins, 2014). Analogous to the mechanism of yeast high-affinity Fe uptake, the MULTICOPPER FERROXIDASE FOX1 of the green alga C. reinhardtii contributes to cellular $\mathrm{Fe}$ acquisition under $\mathrm{Fe}$ deficiency (La Fontaine et al., 2002).

Bernal et al. (2012) report that severe $\mathrm{Cu}$ deficiency (no added $\mathrm{Cu}$ ) induces Fe deficiency in the Arabidopsis spl7 mutant, and that this is associated with decreased in vitro ferroxidase activity. Moreover, $\mathrm{Cu}$ deficiency causes reduced root-to-shoot Fe translocation, leading to decreased levels of the Fe storage protein ferritin and $\mathrm{Fe}$-dependent catalase activity in shoots. Physiological Fe deficiency in shoots of severely $\mathrm{Cu}$-deficient plants activates root Fe deficiency responses, namely increased root surface $\mathrm{Fe}$ (III)-chelate reductase activity and IRT1 transcript levels, in accordance with the known systemic control of some root Fe deficiency responses. Furthermore, under $\mathrm{Cu}$ deficiency conditions but supplemented with extra $\mathrm{Fe}$, the growth rate of the spl7-2 mutant shows a marked improvement, and chlorophyll concentrations are increased to $\sim 85 \%$ of wild-type levels. This is consistent with a model according to which, one or several Fe transmembrane transport processes of plants depend on MCO-mediated ferroxidase activity, similar to yeast, humans and green algae. Indeed, two MCO proteins, LOW PHOSPATE ROOT 1 (LPR1) and LOW PHOSPATE ROOT 2 (LPR2), together with a P5-type ATPase (PDR2), have central roles in local phosphate sensing, and this response is linked to Fe homeostasis (Svistoonoff et al., 2007; Ward et al., 2008; Ticconi et al., 2009; Müller et al., 2015). Phosphate deficiency induces the inhibition of root growth in Arabidopsis seedlings, and it is proposed that this is a consequence of Fe toxicity in the root tip (Ward et al., 2008). Interestingly, the MCO LPR1 functions as a cellwall localized ferroxidase and is responsible for apoplastic Fe(III) deposition in roots tips under phosphate deficiency (Müller et al., 2015). Some authors hypothesize that COPT2 may be a candidate for $\mathrm{Cu}$ transport to LPR1 and LPR2 because roots of copt2 mutants are longer than those of the wild-type, which is in accordance with the phenotype detected in lpr1 and lpr2 mutants (Svistoonoff et al., 2007; Perea-García et al., 2013). Further insights into the molecular basis of cellular Fe export pathways in plants may help to improve the knowledge of the connections between $\mathrm{Cu}$ and $\mathrm{Fe}$ homeostasis in plants, and provide important insights for combating Fe deficiency in crops and for advancing biofortification approaches.

Here, we summarize the current knowledge of the crosstalk between $\mathrm{Cu}$ and $\mathrm{Fe}$ homeostasis and provide a partial characterization of the role of Arabidopsis LACCASE12 (LAC12), which encodes a member of the laccase multicopper oxidase (LMCO) protein family, in root-to-shoot Fe partitioning.

\section{MATERIALS AND METHODS}

\section{Plant Material}

The Arabidopsis thaliana T-DNA insertion lines SALK_004019 (lac12-1, Col-0 background), SALK_047456 (lac12-2, Col-0 background) and SALK_070852 (ao, Col-0 background) were obtained from the Nottingham Arabidopsis Stock Centre, based 
on http://signal.salk.edu. Col-0 seeds were obtained from the NASC. The irt1 mutant (pam42) was obtained from Prof. Dr. Dario Leister, LMU Munich (DE) (Varotto et al., 2002). spl7-2 and $f r d$ 3-7 mutants were previously used in our lab (Bernal et al., 2012; Quintana et al., 2021).

\section{Growth Conditions and Experimental Treatments}

For selection of homozygous mutant lines, plants were cultivated on soil. After 4 to 5 days of seed stratification on moist paper at $4^{\circ} \mathrm{C}$, seeds were transferred onto pre-fertilized soil mixture Type Minitray (Balster Einheitserdewerk, Fröndenberg) in $30 \times 50 \mathrm{~cm}$ plastic square trays. Germination and cultivation were performed in a growth chamber at $20^{\circ} \mathrm{C}, 50 \%$ relative humidity, in long-day conditions ( $16 \mathrm{~h}$ light, $8 \mathrm{~h}$ dark) at a light intensity of $120 \mu \mathrm{mol}$ $\mathrm{m}^{-2} \mathrm{~s}^{-1}$ (Percival CU-41L4; CLF Climatics).

For sterile plant growth, seeds were rinsed in $70 \%(\mathrm{v} / \mathrm{v})$ ethanol for $1 \mathrm{~min}$, surface-sterilized in a solution containing $1.2 \%(\mathrm{w} / \mathrm{v})$ $\mathrm{NaOCl}$ and $0.02 \%(\mathrm{v} / \mathrm{v})$ Triton X-100 for $10 \mathrm{~min}$ and washed five times with ultrapure water (Milli-Q; Merck). Then, seeds were sown on a modified Hoagland's solution containing macro- and micronutrients as described (Bernal et al., 2012), supplemented with $1 \%(\mathrm{w} / \mathrm{v})$ sucrose and solidified with $1 \%(\mathrm{w} / \mathrm{v})$ agar Type $M$ (Sigma) in $12 \times 12 \mathrm{~cm}$ square Petri plates (Greiner Bio-One). Plates were placed at $4^{\circ} \mathrm{C}$ in the dark for $3 \mathrm{~d}$ and then grown vertically for 10 days in $11 \mathrm{~h}$ light, $22^{\circ} \mathrm{C} / 13 \mathrm{~h}$ dark, $18^{\circ} \mathrm{C}$ cycles (Percival CU-41L4; CLF Climatics). To prepare the treatment Hoagland's medium, Agar M (Sigma) was washed with EDTA to remove all contaminant metals as described (Haydon et al., 2012). Treatment Hoagland media contained 1\% (w/v) sucrose and solidified with $0.8 \%$ (w/v) EDTA-washed agar Type M (Sigma). After 10 days of growth, seedlings were transferred to treatment Hoagland media from which Fe was omitted (-Fe treatment) or added as $10 \mu \mathrm{M}$ FeHBED (+Fe treatment) for 5 days. To perform Perls' stain, 7-d-old seedlings were cultivated in the same conditions described above but were sown directly on the treatment media.

\section{Quantification of Plant Biomass and Tissue Elemental Concentrations}

Shoots and roots of 15-day-old seedlings were separated with a scalpel, pooled from 20 seedlings, washed in ultrapure water and carefully blotted dry using paper towels to quantify shoot and root fresh biomass. Subsequently, shoots and roots were desorbed in $35 \mathrm{~mL}$ of an ice-cold solution of $5 \mathrm{mM} \mathrm{CaCl}_{2}$ and $1 \mathrm{mM}$ MES-KOH, pH 5.7, for $10 \mathrm{~min}$, in ice-cold solution of $5 \mathrm{mM} \mathrm{CaSO}_{4}, 10 \mathrm{mM} \mathrm{Na}$ EDTA, pH 5.7 for $5 \mathrm{~min}$, and twice in ice-cold ultrapure water for $1 \mathrm{~min}$. Shoot and root tissues were dried at $60^{\circ} \mathrm{C}$ for $3 \mathrm{~d}$ and equilibrated at room temperature for at least $3 \mathrm{~d}$ before homogenization. Dried shoot and root material was homogenized, weighed, and subsequently digested for the quantification of elemental concentrations by inductively coupled plasma atomic emission spectrometry (ICPOES) in an iCAP 6500 DUO instrument (Thermo-Fisher) as described previously (Sinclair et al., 2017). Three independent experiments were carried out, each comprising 4 to 5 plates per genotype and treatment.

\section{Genotyping}

Plants homozygous for the lac12 T-DNA insertions were identified by PCR on genomic DNA: The specific primer for the left border of the T-DNA (5'-GCGTGGACCGCTTGC TGCAACT- $\left.3^{\prime}\right)$ was used in combination with a LAC12 specific primer (5'- CACCATGACGACTGTTCACACATTCTCT-3 $\left.{ }^{\prime}\right)$ for SALK_004019 (lac12-1) and for SALK_047456 (lac122), respectively. LAC12 WT allele was identified by PCR on genomic DNA using the following LAC12 specific primers, LAC12_F 5'-CACCATGACGACTGTTCACACATTCTCT-3' and LAC12-R 5'-CTAGCAAATAGGTAGATCGTGAGGA-3'. Plants homozygous for the ao T-DNA insertions were identified by PCR on genomic DNA: The specific primer for the left border of the T-DNA ( $5^{\prime}$-GCGTGGACCGCTTGCTGCAACT$\left.3^{\prime}\right)$ was used in combination with an $A O$ specific primer ( $5^{\prime}$ - CACCATGATGAGACCGAAGAGATCA $-3^{\prime}$ ) for SALK_070852. AO WT allele was identified by PCR on genomic DNA using the following $A O$ specific primers, AO-F $5^{\prime}$ - CACCATGATGAGACCGAAGAGATCA $-3^{\prime}$ and AO-R 5'-TCAGCGTTTAGTCTGACCACATCC -3'. Genomic DNA isolation was done as described (Edwards et al., 1991).

\section{RNA Extraction, cDNA Synthesis, and Real-Time PCR}

Total RNA was isolated from 100-mg subsamples of frozen root and shoot tissues exactly as described (Bernal et al., 2012). Quality and quantity of RNA was analyzed spectrophotometrically at the wavelengths of 260 and $280 \mathrm{~nm}$, respectively. Equal amounts of RNA ( $1 \mu \mathrm{g})$ were used as a template for cDNA synthesis (RevertAid First Strand cDNA Synthesis Kit, Thermo Fisher Scientific) with oligo-dT primers following manufacturer's instructions. RT-qPCR was performed on a LightCycler480 (Roche) in a $10-\mu \mathrm{L}$ reaction mixture containing $16 \mathrm{ng}$ of cDNA, each primer at $0.25 \mu \mathrm{M}$ and $5 \mu \mathrm{L}$ of $2 \mathrm{X}$ GoTaq qPCR Master Mix (Promega) as described (Quintana et al., 2021). Reaction efficiencies (RE) for each PCR reaction were determined with the LinRegPCR program, version 2016.0 (Ruijter et al., 2009). Threshold cycle values were calculated for each reaction at a threshold value of the normalized reporter $R_{\mathrm{n}}$ of 0.2 . Relative transcript levels were calculated as follows: $\mathrm{RTL}=1000 \times \mathrm{RE}_{\mathrm{m}}-\Delta C T$, whereby $\mathrm{RE}_{\mathrm{m}}$ is the arithmetic mean of the reaction efficiency and $\Delta C_{T}$ values were calculated as follows: $\Delta \mathrm{C}_{T}=\mathrm{C}_{\mathrm{T}}$ (target gene) $-\mathrm{C}_{\mathrm{T}}$ (constitutively expressed reference gene: $U B Q 10)$. Primers sequences are listed in Supplementary Table 1.

\section{Yeast Constructs, Strains, and Growth}

IRT1 and LAC12 cDNAs, including translational start and stop codon, were amplified from wild-type cDNA by RT-PCR with IRT1-ATG_F 5'-CACCATGGCTTCAAATTCAGCAC-3' and IRT1-cDNA_TAA_R 5'-TTAAGCCCATTTGGCGATAATCG-3' and with LAC12-ATG_F 5'-CACCATGACGACTGTTCACACA TTCTCT-3'; LAC12-TAG-R 5'-CTAGCAAATAGGTAGATC 
GTGAGGA-3, respectively. Both cDNAs were cloned into pENTR-D/TOPO (Invitrogen) and verified by Sanger sequencing before performing the recombination reaction into the yeast expression vector pFL61-Gateway (Desbrosses-Fonrouge et al., 2005) using LR clonase (Invitrogen) according to manufacturer's protocol. Both constructs were checked by Sanger sequencing. For complementation assays, competent $S$. cerevisiae yeast cells of the wild-type (WT, DY1457) and fet3fet4 mutant (DEY1453; Dix et al., 1994) strains were transformed with $1 \mu \mathrm{g}$ of each construct or the empty vector (Dohmen et al., 1991). The corresponding WT yeast strain transformed with the empty vector was used as positive control. Transformants were selected on SD media lacking uracil (SD-Ura) pH 5.7 with 2\% (w/v) D-glucose as a carbon source. For each construct, three independent transformant colonies were grown overnight at $30^{\circ} \mathrm{C}$ in $2 \mathrm{~mL}$ SD-Ura pH 5.7 media to early stationary phase $\left(\mathrm{OD}_{600} \approx 0.3, c a\right.$. $10^{7}$ cells $\left.\mathrm{ml}^{-1}\right)$. Yeast cells were then centrifuged at $13.000 \mathrm{rpm}$ for 1 min, washed once with SD-Ura $\mathrm{pH} 5.7$ media with $2 \%$ $(\mathrm{w} / \mathrm{v}) \mathrm{D}$-glucose and resuspended in the same media. Aliquots of cell suspensions were serially diluted with SD-Ura pH 5.7 media with $2 \%(\mathrm{w} / \mathrm{v}) \mathrm{D}$-glucose in a 10 -fold series ( $\mathrm{ca} \cdot 10^{7}$, $10^{6}, 10^{5}$, and $10^{4}$ cells $\mathrm{ml}^{-1}$, based on $\left.\mathrm{OD}_{600}\right)$. Drops of $10 \mu \mathrm{L}$ were spotted onto solid ( $2 \% \mathrm{w} / \mathrm{v}$ Bacto agar, Difco) SD-Ura $\mathrm{pH} 5.7$ media with $2 \%(\mathrm{w} / \mathrm{v})$ D-glucose supplemented with or without $0.5 \mathrm{mM} \mathrm{FeSO}$. Plates were incubated at $30^{\circ} \mathrm{C}$ for 3 days and photographed using a Nikon Digital SLR camera with an AF-S DX Zoom NIKKOR 18-70 mm 1:3, 5-4,5G ED-IF objective.

\section{Localization of Fe(III) With Perls' Stain}

7-d-old seedlings germinated and grown on Fe-sufficient (+Fe, $10 \mu \mathrm{M}$ FeHBED) or Fe-deficient (-Fe, $0 \mu \mathrm{M}$ FeHBED) Hoagland's medium solidified with EDTA-washed agar were harvested at ZT1. Roots $(\sim 4 \mathrm{~cm}$ from root tip) of 3 to 5 seedlings were pooled and washed once with ice-cold $10 \mathrm{mM}$ EDTA (pH 5.7) for $5 \mathrm{~min}$ and three times with ice-cold ultrapure water for $1 \mathrm{~min}$. Then, the samples were vacuum-infiltrated with Perls' stain solution (equal volumes of $4 \%(\mathrm{v} / \mathrm{v}) \mathrm{HCl}$ and $4 \%(\mathrm{w} / \mathrm{v})$ potassium ferrocyanide) for 15 min (Roschzttardtz et al., 2009). Roots were incubated in the Perls' stain solution for another $15 \mathrm{~min}$ at room temperature and then rinsed three times with ultrapure water and mounted in ultrapure water for visualization. DAB intensification was performed as described (Brumbarova and Ivanov, 2014). Photographs were taken using an Axioscope microscope, Axiovision sofware (Zeiss) and Axiocam MRc Rev. 3 camera.

\section{In-gel Detection of Ferroxidase and Phenoloxidase Activities}

These assays were performed exactly as described (Bernal et al., 2012). The experiment was repeated twice using independent protein samples extracted from aliquots of frozen homogenized tissues harvested in a common experiment. Band quantification was done using the ImageJ software.

\section{Measurement of Chlorophyll Concentrations}

Total chlorophyll was extracted with $1.5 \mathrm{ml}$ methanol from 20 to $40 \mathrm{mg}$ of frozen ground shoot tissues of 15 -day-old seedlings. Absorbance values were determined spectrophotometrically at the wavelengths of 652 and $665 \mathrm{~nm}$ in 96-well plates in a Synergy HTX Multi-Mode Reader (Agilent, former BioTek), using methanol as a blank. Microplate path length-correction to $1 \mathrm{~cm}$ was carried out with the factor 0.51 according to Warren, 2008. Chlorophyll concentrations were calculated as described (Porra et al., 1989) and normalized to fresh biomass.

\section{In silico Sequence Analysis of MCO in Arabidopsis thaliana}

Search of the TAIR10 protein databases was done with the BLAST programme (Altschul and Lipman, 1990) using the previously identified Saccharomyces cerevisiae FET3 protein sequence, the multicopper ferroxidase (MCO) involved in high-affinity $\mathrm{Fe}$ uptake. Alignments were done using Mafft program (Katoh et al., 2002). The location of putative transmembrane helices was predicted using the programme ARAMEMNON at http:// aramemnon.botanik.uni-koelm.de/ (Schwacke et al., 2003).

\section{D-Structure Modeling}

To model the 3D-structure of LAC12, LAC13 and AO from Arabidopsis thaliana, the Phyre2 version 2.0 was used (Kelley and Sternberg, 2009) and subsequently compared with the well-known 3D-structure of ScFET3 (Taylor et al., 2005). LAC12, LAC13 and AO 3D-structure models were submitted to the 3DLigandSite server to predict in silico metal binding (Wass et al., 2010).

\section{Statistical Analysis}

Multiple comparisons were conducted by two-way analysis of variance (ANOVA; Tukey's honestly significant difference $[\mathrm{HSD}]$ ) using Statgraphics software (version XV.I; Statpoint Technologies).

\section{RESULTS}

\section{In silico Identification of FET3 Homologs in Arabidopsis thaliana}

In humans, membrane-associated caeruloplasmin, a multicopper oxidase, drives $\mathrm{Fe}$ export from cells via the $\mathrm{Fe}^{2+}$ exporter ferroportin by oxidizing $\mathrm{Fe}^{2+}$ upon its arrival on the external face of the plasma membrane (Hellman and Gitlin, 2002). Previously, we observed that severely Cu-deficient plants are also Fe-deficient as a result of impaired root-toshoot Fe partitioning (Bernal et al., 2012). We speculated on a possible role of a MCO in the cellular export of $\mathrm{Fe}$ into xylem vessels in roots of $A$. thaliana, in analogy with membrane-associated human caeruloplasmin. Therefore, we conducted an in silico analysis to identify candidate MCOs functioning as ferroxidases based on the best studied MCO FET3 of the high-affinity Fe uptake system of Saccharomyces 
cerevisiae (Askwith et al., 1994). FET3 has four catalytic Cu ions in the mononuclear $\mathrm{Cu}$ cluster (T1) and the trinuclear $\mathrm{Cu}$ cluster (T2/T3), where the oxidation of $\mathrm{Fe}^{2+}$ cations takes place. Based on site-directed mutagenesis of the $S$. cerevisae FET3 protein, previous research identified D319 and D320 to be critical for the growth of yeast under Fe-deficient conditions. In particular, mutation of D320 abrogated the FET3-dependent Fe uptake function (Bonaccorsi di Patti et al., 2005; Quintanar et al., 2007). Kinetic studies of mutations of the E185, D283, and D409 residues, respectively, showed their important role in $\mathrm{Fe}$ (II) oxidation (Quintanar et al., 2007). In LPR1 and LPR2, two MCOs that act as ferroxidases mediating root phosphate deficiency responses in Arabidopsis, the acidic residues of FET3 are also conserved (E269, D370 and D462 in LPR1 and E271, D372 and D464 in LPR2) as shown the structural superimpositions of LPR1 and LPR2 onto FET3, respectively (Müller et al., 2015). As a first step, a BLAST search using the yeast FET3 sequence generated a list of candidate MCOs that may have ferroxidase functions in A. thaliana, among them the LMCO family and several ascorbate oxidases (Table 2). An alignment of the LMCO, the ascorbate oxidase $\mathrm{AO}$ and the LPR1 and LPR2 sequences from A. thaliana with the sequence of FET3 from yeast revealed the conserved $\mathrm{C}$ and $\mathrm{H}$ residues that form the trinuclear (T2/T3) and mononuclear (T1) Cu binding sites (Supplementary Figure 1). The essential ferroxidase E185, D283, and D409 residues of yeast FET3, seem to be conserved in LAC3 (E219, D333, D462), LAC5 (E220, D341, D472) LAC12 (E218, D326, D457), LAC13 (E217, D331, D461), LPR1 (E269, D370, and D462), LPR2 (E271, D372, and D464) and AO (E198, D354, and D465) (Supplementary Figure 1, residues highlighted in pink), and the ferroxidase aspartic residues D319 and D320 of FET3 appear to be conserved in LAC8 (D407, D408), LAC9 (D410, D411), LAC12 (D351, D352) and LAC16 (D367, D368) (Supplementary Figure 1, residues highlighted in red). This conservation was suggested based on our BLAST results and alignment, as well as on the comparison of the $3 \mathrm{D}$ structure of FET3 with the 3D-structure models of LAC12, LAC13, and AO (Supplementary Figure 2A). Our in silico analysis was also compared with the homology modeling previously conducted for LPR1 and LPR2 and the structural superimpositions of LPR1 and LPR2 onto FET3 that allowed to assign the ferroxidase residues in Arabidopsis (Müller et al., 2015). The two laccase multicopper oxidase proteins (LMCOs) LAC12 (At5g05390) and LAC13 (At5g07130), and AO (At4g39830), a MCO-ascorbate oxidase of the cupredoxin superfamily, were among the Arabidopsis proteins showing highest similarity $(42 \%, 41 \%$, and $43 \%$, respectively) to FET3 (Table 2). In addition, LAC12 and AO also contained one predicted transmembrane domain as FET3 (Supplementary Figures 1, 2B) (McCaig et al., 2005; Turlapati et al., 2011; Santamaría et al., 2018). According to the eFP browser, LAC12 and $L A C 13$ were expressed mainly in the vasculature of the root maturation zone adjacent to the xylem (Brady et al., 2007; Winter et al., 2007) (Supplementary Figure 3A).

\section{LAC12 Is Upregulated Under Fe Deficiency in Wild-Type Seedlings}

To examine if LAC12 expression was regulated under $\mathrm{Fe}$ deficiency, we cultivated wild-type (WT) and irt1 seedlings under Fe-deficient and Fe-sufficient (control) conditions for $10-\mathrm{d}$, subsequent to a 5 - $\mathrm{d}$ pre-cultivation period on $\mathrm{Fe}$ sufficient medium (Figure 1; Quintana et al., 2021). The IRON REGULATED TRANSPORTER1 (irt1) mutant defective in root high-affinity $\mathrm{Fe}$ uptake was included as a control genotype exhibiting constitutive activation of the Fe deficiency responses (Connolly et al., 2002; Henriques et al., 2002; Varotto et al., 2002; Vert et al., 2002; Kobayashi and Nishizawa, 2012). Root LAC12 transcript levels were 3.5 to 4 -fold elevated under Fe deficiency conditions compared to control conditions in the wildtype, and 24 -fold elevated in the irt 1 mutant. Taking into account these results, we decided to characterize the multi-copper oxidase (MCOs) LAC12 as a candidate ferroxidase.

\section{A LAC12 cDNA Complements the Fe Uptake-Defective Yeast fet3fet4 Mutant}

We tested whether heterologous expression of the LAC12 coding sequence complements the Fe uptake-defective $S$. cerevisiae fet3fet4 mutant (Figure 2). Expression of LAC12 significantly

TABLE 2 | Putative candidate genes for ferroxidase functions identified through in silico analysis.

\begin{tabular}{|c|c|c|c|c|c|c|}
\hline AGI code & Name & Description & $\begin{array}{c}\text { TM } \\
\text { domain }\end{array}$ & $\begin{array}{l}\text { Fe-uptake-like D } \\
\text { residues }^{1}\end{array}$ & $\begin{array}{c}\% \text { Homology with } \\
\text { ScFET3 }\end{array}$ & Publication \\
\hline AT5G21105 & None & Plant L-ascorbate oxidase & 1 & Yes & 41 & Unpublished \\
\hline AT4G39830 & $\begin{array}{l}\text { Ascorbate } \\
\text { oxidase } \mathrm{AO}\end{array}$ & $\begin{array}{c}\text { Cupredoxin superfamily } \\
\text { protein }\end{array}$ & 1 & Yes & 43 & Santamaría et al., 2018 \\
\hline AT5G21100 & None & Plant L-ascorbate oxidase & 1 & Yes & 42 & Unknown \\
\hline AT2G30210 & $\begin{array}{l}\text { Laccase } 3 \\
\text { (LAC3) }\end{array}$ & $\begin{array}{l}\text { Member of laccase family } \\
\text { of genes }\end{array}$ & 1 & Yes & 42 & $\begin{array}{l}\text { Rojas-Murcia et al., 2020; } \\
\text { Zhuang et al., } 2020\end{array}$ \\
\hline AT5G05390 & $\begin{array}{l}\text { Laccase } 12 \\
\text { (LAC12) }\end{array}$ & $\begin{array}{c}\text { Member of laccase family } \\
\text { of genes }\end{array}$ & 1 & Yes & 42 & Carrió-Seguí et al., 2019b \\
\hline AT5G07130 & $\begin{array}{l}\text { Laccase } 13 \\
\text { (LAC13) }\end{array}$ & $\begin{array}{c}\text { Member of laccase family } \\
\text { of genes }\end{array}$ & 1 & Yes & 41 & $\begin{array}{l}\text { Carrió-Seguí et al., 2019b; } \\
\text { Rojas-Murcia et al., } 2020\end{array}$ \\
\hline
\end{tabular}

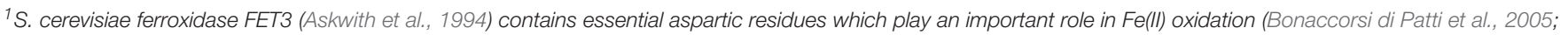
Quintanar et al., 2007). 


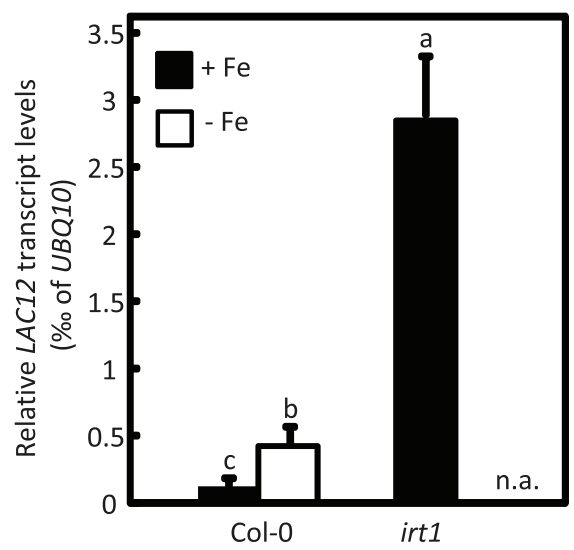

FIGURE 1 | LAC12 transcript levels increase under Fe deficiency. Relative LAC12 transcript levels in roots of wild-type (Col-0) and irt1 seedlings are analyzed by RT-qPCR. Seedlings were cultivated in standard Hoagland's medium for $10 \mathrm{~d}$ (5 $\mu \mathrm{M}$ FeHBED; unwashed agar) followed by a growth period of $5 \mathrm{~d}$ on Fe-sufficient (+Fe, $10 \mu \mathrm{M}$ FeHBED) and Fe-deficient (-Fe, $0 \mu \mathrm{M}$ FeHBED) agar-solidified Hoagland's medium (EDTA-washed agar), on vertically oriented petri plates. Bars show arithmetic means $\pm \mathrm{SD}$ of relative transcript levels normalized to UBQ10, and were calculated from $n=3$ technical replicates from one experiment representative of four independent biological experiments. n.a. not analyzed. Different characters denote statistically significant differences $(P<0.05)$ between means based on ANOVA (Tukey's HSD).

improved fet3fet 4 mutant growth compared with the mutant strain transformed with empty vector on ordinary SD medium (Figure 2A), similar to yeast fet3fet4 cells expressing the IRT1 cDNA as a positive control (Eide et al., 1996). Both the fet 3 fet 4 mutant and all yeast transformants were able to grow equally well on medium supplemented with $0.5 \mathrm{mM}$ Fe (Figure 2B). This result suggests that in yeast, LAC12 may be able to function as a multicopper oxidase with ferroxidase activity, which would be sufficient to complement fet 3 fet 4 .

\section{Arabidopsis lac12 Mutants Are Sensitive to Fe Deficiency}

To further investigate the role of A. thaliana LAC12, two independent T-DNA insertion lines disrrupted in the LAC12 gene were identified in the Columbia-0 (Col-0) background and characterized (see Materials and Methods). The T-DNAs insertions were confirmed in the 4th exon (lac12-1) and 5th exon (lac12-2) of the LAC12 gene, respectively (Supplementary Figures 3B,C). LAC12 transcript levels were reduced in both lac12 T-DNA insertion lines (Supplementary Figure 3E). To examine if Fe deficiency affects the growth of lac12 mutant seedlings, wild-type and lac12 mutant seedlings were cultivated in Fe-deficient and Fe-sufficient agar-solidified media (Haydon et al., 2012). Under Fe-sufficient conditions, the appearance, biomass and leaf chlorophyll concentrations were similar in wild-type seedlings and the two lac12 mutant lines (Figure 3). On Fe-deficient media, characteristic symptoms of Fe deficiency were detected in the wild-type (Marschner, 1995) including chlorosis, reduced root and shoot biomass and decreased leaf chlorophyll concentrations. Compared to wild-type seedlings, both lac12 mutant lines were more sensitive to Fe deficiency, with more severely reduced biomass and chlorophyll concentrations (Figure 3). These data suggest that the multicopper oxidase LAC12 is required for wild-type levels of plant growth under $\mathrm{Fe}$ deficiency. This means that LAC12 function seems to be necessary to allow plants to behave as wild-type plants under Fe deficiency.

\section{Elevated Root Fe Accumulation and Decreased Shoot Total Fe in Arabidopsis lac12 Mutants}

To test whether Fe levels in plant tissue are altered alongside the enhanced sensitivity to Fe deficiency of lac12 mutants, we quantified metal concentrations by inductively coupled plasma optical emission spectrometry (ICP-OES) in shoots
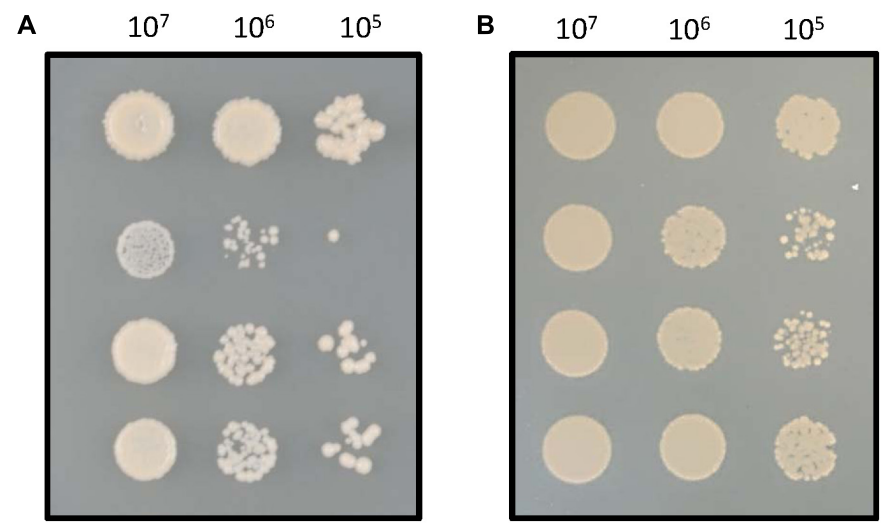

$\mathrm{WT}+\mathrm{eV}$

fet3fet $4+\mathrm{eV}$

fet3fet4 + IRT1

fet3fet4 + LAC12

FIGURE 2 | Heterologous expression of the AtLAC12 cDNA complements a Fe uptake-defective fet3fet4 mutant of Saccharomyces cerevisiae. Wild-type and the Fe uptake-defective fet3fet4 mutant of Saccharomyces cerevisiae transformed with the empty vector pFL61 Gateway (eV) or expressing IRT1 or LAC12 cDNAs of

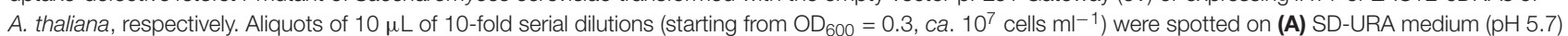
and (B) SD-URA medium ( $\mathrm{pH}$ 5.7) supplemented with $0.5 \mathrm{mM} \mathrm{FeSO}_{4}$. Images are representative of three independent transformant colonies from each of two independent experiments. 

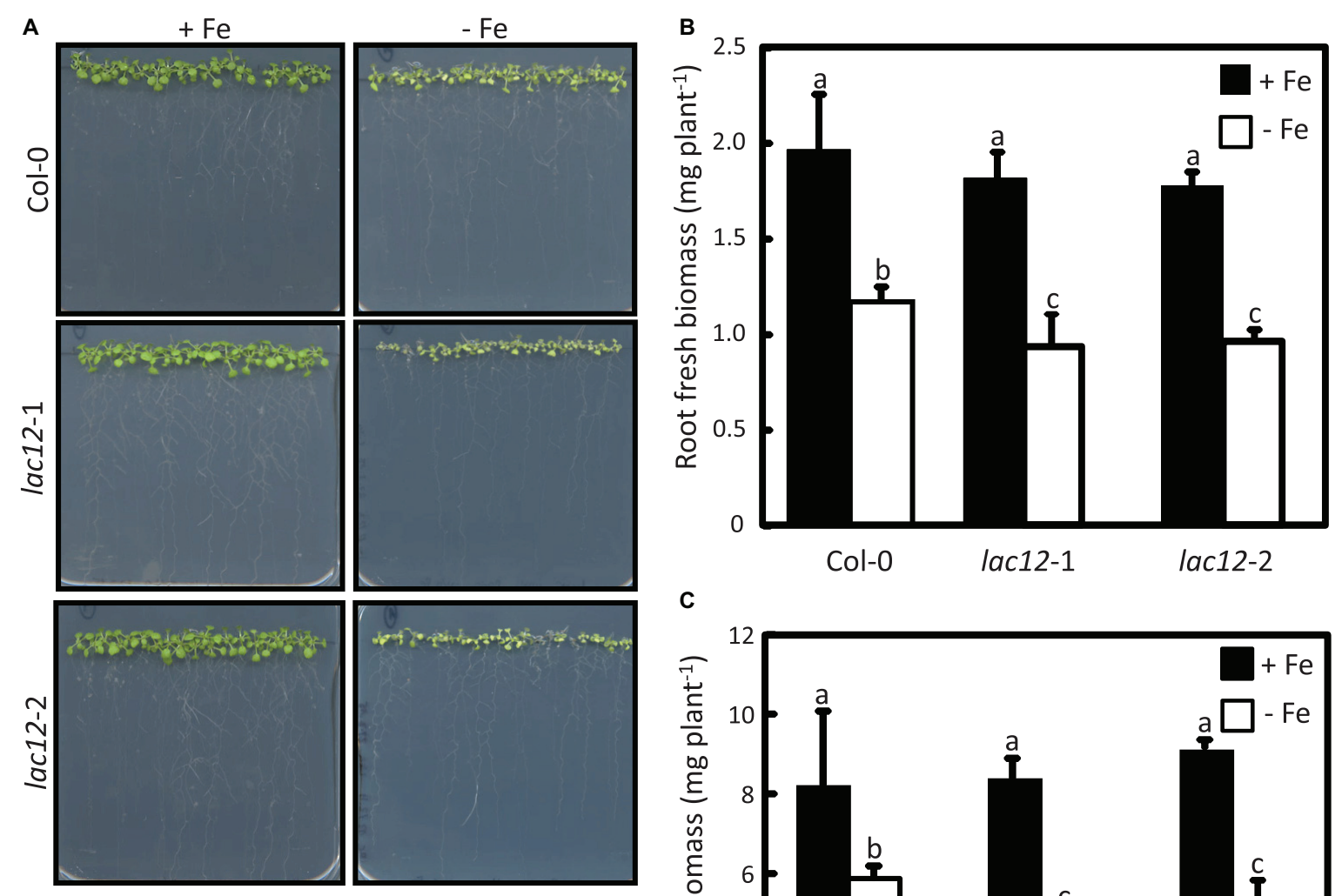

C
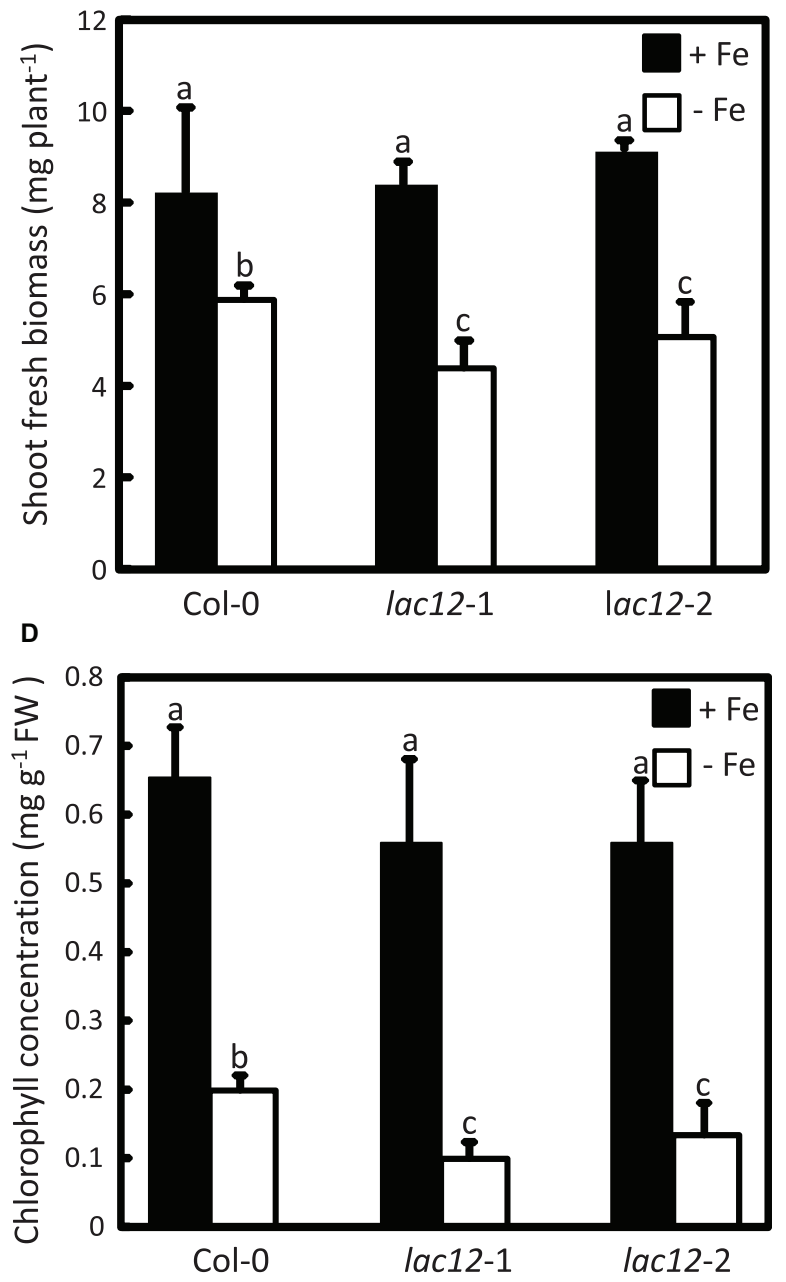

FIGURE 3 | Arabidopsis lac12 mutants are more sensitive to Fe deficiency than the wild-type. (A) Photographs of 15-day-old seedlings of WT, lac12-1 and lac12-2 mutant lines grown in standard Hoagland's medium for $10 \mathrm{~d}$ ( $5 \mu \mathrm{M}$ FeHBED; unwashed agar) followed by a growth period of $5 \mathrm{~d}$ on Fe-sufficient (+Fe, $10 \mu \mathrm{M}$ FeHBED) and Fe-deficient (-Fe, $0 \mu \mathrm{M}$ FeHBED) agar-solidified Hoagland's medium (EDTA-washed agar), on vertically oriented petri plates. Photographs are from one experiment representative of three independent experiments. Fresh biomass of (B) roots and (C) shoots, and (B) leaf chlorophyll content of WT, lac12-1 and lac12-2 seedlings grown on vertically oriented petri plates as described above. Bars show arithmetic means \pm SD of three replicate plates, with 20 seedlings per plate, from one experiment representative of three independent experiments. Each replicate consisted of pooled material of 20 seedlings from one plate (B and $\mathbf{C})$, or 5 seedlings per replicate plate (D). Different characters denote statistically significant differences $(P<0.05)$ between means based on ANOVA (Tukey's HSD). 
and roots of seedlings cultivated in Fe-sufficient and Fedeficient agar-solidified media (Figure 4). Upon cultivation in control conditions, root (Figure 4A) and shoot (Figure 4B) Fe concentrations in wild-type and lac12 mutant seedlings were mostly equivalent, except for lac12-2 in which Fe concentrations in roots were lower compared to wild-type and lac12-1, respectively. However, on Fe-deficient media, root $\mathrm{Fe}$ concentrations of lac12 mutants were between 20\% (lac12-1) and $30 \%$ (lac12-2) elevated compared to wild-type seedlings. Total shoot Fe content was between 40\% (lac12-1) and 25\% (lac12-2) lower in lac12 mutants than in the wild-type under Fe deficiency conditions (Figure 4C). Shoot Fe concentrations of these plants were very low and similar between wild-type and lac12 mutants, consistent with growth limitation by Fe.

Increased Fe concentrations in roots of lac12 mutant seedlings compared to wild-type roots upon cultivation under Fe deficiency prompted us to use Perls' stain for Fe localization, without and with DAB intensification (Figures 5A,B and Supplementary Figure 5). Perls' stain detected Fe in the vascular tissue in lac12 mutant roots grown under Fe deficiency, but not in wild-type roots (Figures 5A,B). The signal was weak and appeared to be present primarily in the stele, this result was additionally supported by combining Perls' stain with the DAB intensification step (Figure 5B). Upon cultivation in control conditions, no Perls' stain was detected in either wild-type nor lac12 mutants. This result is counter-intuitive because in all lines analyzed the $\mathrm{Fe}$ concentrations in control conditions were higher than in $\mathrm{Fe}$ deficient conditions. A possible explanation may lie in a different Fe distribution across the root, whereby Fe may usually be widely distributed among the entire root and thus not detectable by Perls' stain, with a more highly localized Fe accumulation in lac12 mutant seedlings grown under Fe-deficient conditions. This result is in full agreement with the strong Fe localization observed in the stele in frd3-7 and spl7-2 mutants, in which root-to-shoot Fe partitioning is impaired (Figure 5C) (Green and Rogers, 2004; Bernal et al., 2012). Taken together, our data suggest that the disruption of LAC12 interferes with root-to-shoot Fe partitioning under Fe deficiency.

Irrespective of their genotype, $\mathrm{Cu}$ concentrations were higher in plants grown under Fe deficiency compared to Fesufficient conditions (Supplementary Figure 6), as previously reported (Waters et al., 2012). Root Mn concentration of wildtype and lac12 mutant plants are higher compared to shoot Mn concentrations in Fe-sufficient conditions (Supplementary Figure 6). Under Fe deficiency, Mn concentrations in both roots and shoots of wild-type and lac12 mutant plants are higher than in Fe-sufficient conditions, in which the increase of root Mn concentration in lac12 mutants were significantly higher when compared to wild-type plants. This result could be related to an increase of IRT1 protein levels, which is well known to transport Mn as well (Eide et al., 1996; Rogers et al., 2000), as is known to happen in Fe-deficient wild-type plants (Quintana et al., 2021). The accumulation of $\mathrm{Zn}$ under Fe deficiency has been previously reported in WT plants (Buckhout et al., 2009) and could be due to a low specificity of IRT1,
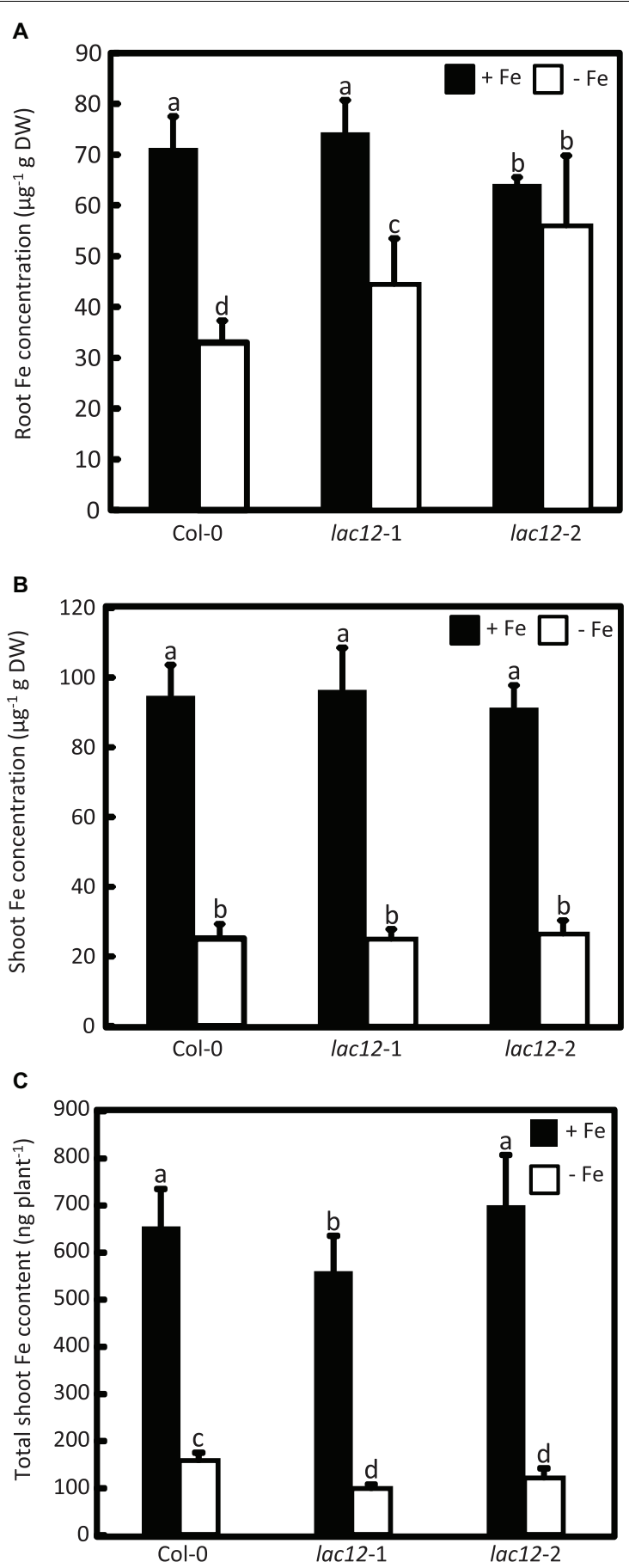

FIGURE 4 | Arabidopsis lac12 mutants accumulate higher Fe concentrations in roots and contain lower total shoot Fe than the wild-type when cultivated under Fe deficiency. Fe concentrations in (A) roots and (B) shoots, and (C) total Fe content in shoots, of WT, lac12-1 and lac12-2 seedlings grown on vertically oriented petri plates as described in Figure 3. Values are arithmetic means $\pm \mathrm{SD}$ of three biological replicate plates from one experiment representative of three independent biological experiments. Metals were quantified in pooled tissues from 15 seedlings per replicate plate. Different characters denote statistically significant differences $(P<0.05)$ between genotypes and Fe treatments based on ANOVA (Tukey's HSD). 


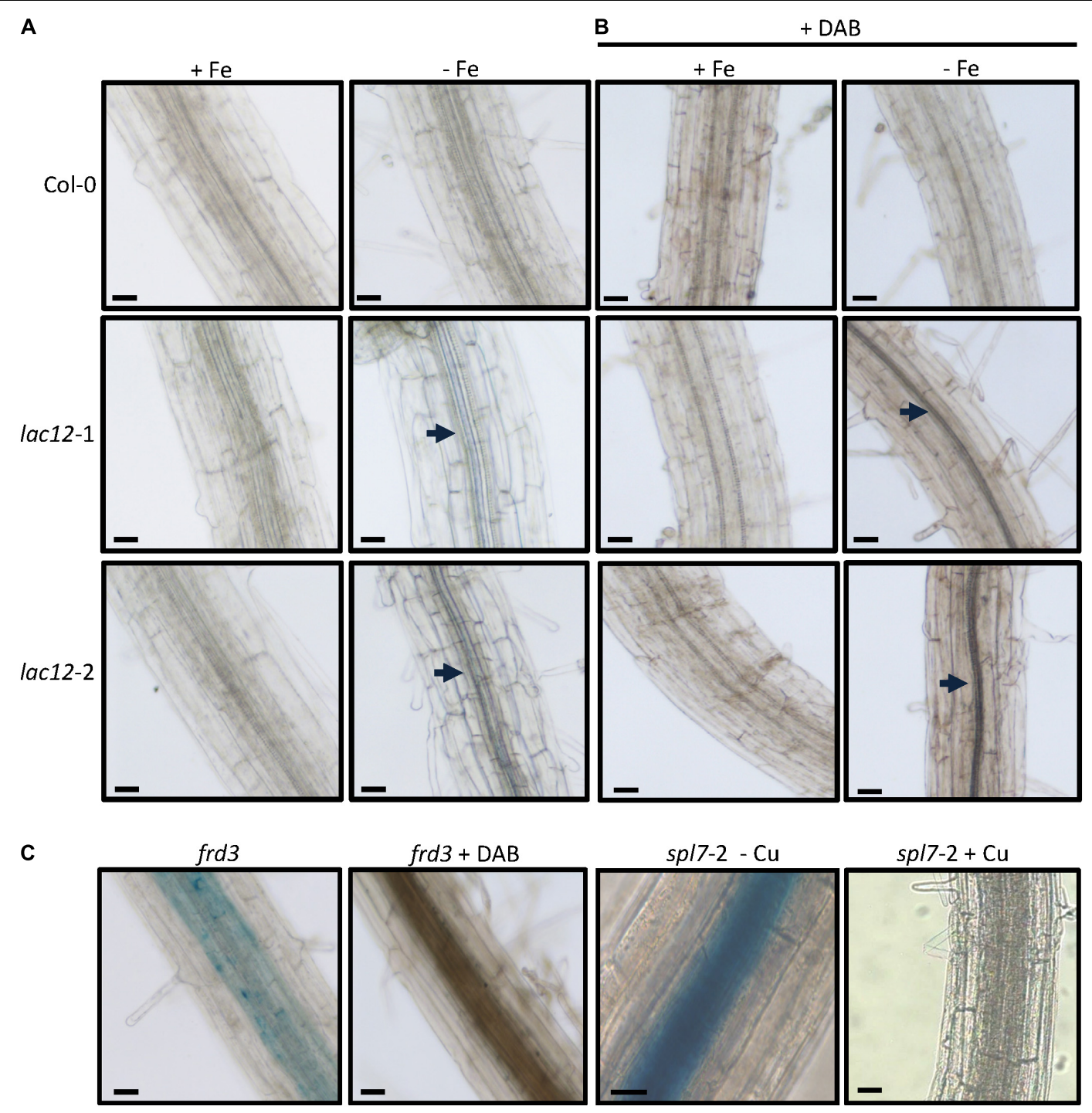

FIGURE 5 | Fe localization in roots of lac12 mutants. (A,B) Histochemical detection of Fe(III) by Perls' blue stain in the root maturation zone of 7-d-old WT, lac12-1 and lac12-2 seedlings grown on Fe-sufficient (+Fe, $10 \mu \mathrm{M}$ FeHBED) or Fe-deficient (-Fe, $0 \mu \mathrm{M}$ FeHBED) agar-solidified Hoagland's medium (EDTA-washed agar) without (A) or with DAB intensification (B). (C) Histochemical detection of Fe(III) by Perls' blue stain in the root maturation zone of frd3 and sp/7-2 seedlings. The frd3 mutant seedlings were grown only under Fe-sufficient conditions for 7 days. Images are of 21 -d-old sp/7-2 seedlings grown on vertical glass plates containing EDTA-washed agar-solidified Hoagland's medium $\left(0.5 \mu \mathrm{M} \mathrm{CuSO}_{4}\right.$, control) or the same medium containing no added copper (-Cu) as described (Bernal et al., 2012). Photographs are representative of $n=10$ to 12 roots stained and imaged in one experiment. Photographs are from one experiment representative of two independent experiments. Arrows point to Perls' stain-positive regions. Scale bars: $50 \mu \mathrm{m}$.

which is transcriptionally induced under Fe-deficient conditions (Supplementary Figure 6) (Vert et al., 2002).

\section{Decreased Ferroxidase and Phenoloxidase Activities in lac12 Mutant Plants}

The functional complementation of the fet3fet4 yeast double mutant with LAC12 (Figure 2) prompted us to further analyze the existence of MCO-related enzyme activities in WT and lac12 mutant plants and its regulation by Fe deficiency (Figure 6). We observed that phenoloxidase and ferroxidase activities in root crude extracts run similarly in denaturing SDS-PAGE protein gels, suggesting that one or several MCO proteins may have, additionally, ferroxidase activity. It is worth mentioning at this point that the in-gel phenoloxidase activity assay detected two bands of activity in all root samples tested, however, we only discuss further the abundance of the upper band because this is the one that runs at a similar position as the band exhibiting ferroxidase activity. Wild-type roots showed an increase in phenoloxidase activity under Fe-deficient conditions. In roots of lac12 mutants, this phenoloxidase activity was lower than in wild-type under both Fe-sufficient conditions and Fe-deficient conditions (Figure 6A). Ferroxidase activity was 


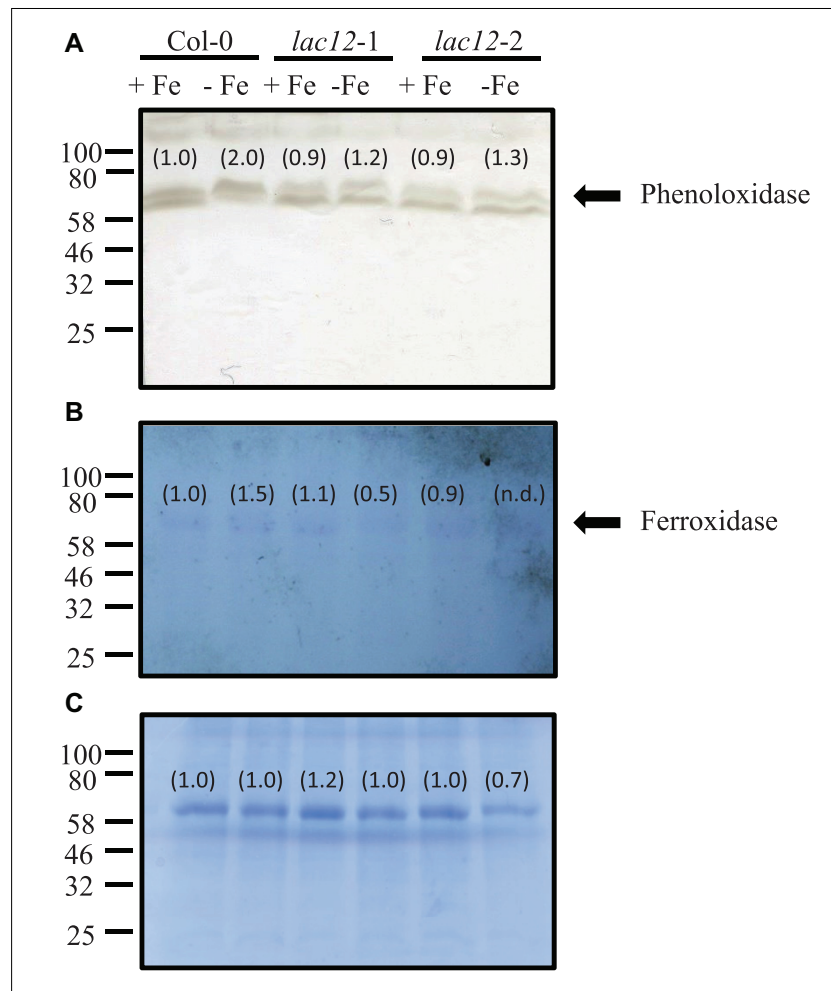

FIGURE 6 | In-ge/ phenoloxidase and ferroxidase activities in lac12 mutants. In-gel detection of phenoloxidase (A) and ferroxidase (B) activities in total protein extracts from roots of WT, lac12-1 and lac12-2 seedlings grown on vertically oriented petri plates as described in Figure 3. Thirty micrograms of protein were loaded per lane. Data shown are from one single independent experiment. (C) Coomassie blue staining to visualize protein loading.

detectable in all root samples and increased slightly under $\mathrm{Fe}$ deficient conditions in wild-type roots (Figure 6B). In lac12 roots, ferroxidase activities were similar to wild-type roots under Fe-sufficient conditions, but decreased under Fe-deficient conditions (Figure 6A). Taken together, under Fe deficiency when LAC12 transcript levels are elevated in the wild-type, decreased activities of both phenoloxidase and ferroxidase in lac12 mutants suggest that LAC12 can act as a ferroxidase in Arabidopsis roots.

\section{Characterization of the Fe Deficiency Responses in lac12 Plants}

To further examine Fe status in lac12 mutants, preliminary data regarding the regulation of the transcript levels of a subset of well-known Fe deficiency response genes were obtained by RT-qPCR in the wild-type and lac12 mutants grown under Fe-sufficient and Fe-deficient conditions (Figure 7). FRO2 and IRT1 transcript levels were strongly increased in roots and bHLH39 was strongly upregulated in roots and shoots under Fe-deficient conditions in both wild-type and lac12 mutant plants. The expression of bHLH39 in Fe-deficient lac12 roots was higher than in Fe-deficient wild-type roots. This may indicate that the expression of some genes of the $\mathrm{Fe}$ deficiency response machinery is enhanced in the lac12 mutant plants compared to wild-type plants under Fe-deficient conditions. Additionally, COTP2, a known Fedeficiency responsive transcript (Colangelo and Guerinot, 2004) encoding a $\mathrm{Cu}$ transporter, was upregulated in both wildtype and lac12 mutant plants under Fe-deficient conditions. Transcript levels of IRON REGULATED 1/FERROPORTIN 1 and 2 (IREG1/FPN1, IREG2/FPN2) were both increased in Fedeficient wild-type roots compared to controls (Figure 7).

\section{Arabidopsis ao Mutants Are Sensitive to Fe Deficiency}

To elucidate whether other genes may account for the $\mathrm{Cu}$ requirement in plant $\mathrm{Fe}$ homeostasis, a preliminary characterization of the ASCORBATE OXIDASE (AO) gene function, another promising candidate, was conducted. One T-DNA insertion line disrupted in the $A O$ gene was isolated in the Columbia-0 (Col-0) background and characterized (see Materials and Methods). The T-DNA insertion was confirmed in the 3rd exon of the $A O$ gene (Supplementary Figures 3B,D). To investigate if the growth of the ao mutant line is affected under Fe deficiency, the same physiological experiments were carried out as for lac12 mutants (Supplementary Figure 4). The ao mutant seedlings produced less root and shoot biomass and contained lower chlorophyll concentrations (Supplementary Figures 4A-D). Under Fe deficiency, shoot Fe concentrations were similar in the ao mutant and the wild-type (Supplementary Figure 4E). The data for root $\mathrm{Fe}$ concentrations were not reliable because of insufficient biomass obtained. $\mathrm{Cu}, \mathrm{Zn}$ and $\mathrm{Mn}$ concentrations were elevated in wild-type and ao mutant seedlings grown under Fe-deficient compared to Fe-sufficient conditions (Supplementary Figure 6). Future work will be required to ascertain the relevance of $A O$ in $A$. thaliana under Fe deficiency.

\section{DISCUSSION}

Less than a decade ago, researchers began to address the interactions between $\mathrm{Cu}$ and $\mathrm{Fe}$ homeostasis in plants, including their uptake by roots, storage and movement within the plant as well as regulatory processes and the physiological relevance of possible interactions at the cellular and whole-plant levels. One goal of this report was to highlight recent progress made on the cross-talk among the homeostasis of $\mathrm{Fe}$ and $\mathrm{Cu}$ (for more details see Table 1 and Introduction section). To expand this further, we also report here initial data toward a characterization of the roles of the two genes $L A C 12$ and $A O$ encoding multicopper oxidases $(\mathrm{MCO})$, in the partitioning of Fe between roots and shoots in A. thaliana.

Previously, we observed that severe $\mathrm{Cu}$ deficiency causes Fe deficiency apparently as a result of an impairment of root-to-shoot $\mathrm{Fe}$ translocation, and this was associated with reduced in vitro ferroxidase activity (Bernal et al., 2012). We hypothesized on a role of one or several MCOs in enabling a possible cellular export step of $\mathrm{Fe}$ into the xylem for rootto-shoot $\mathrm{Fe}$ translocation in A. thaliana, similar to what is 

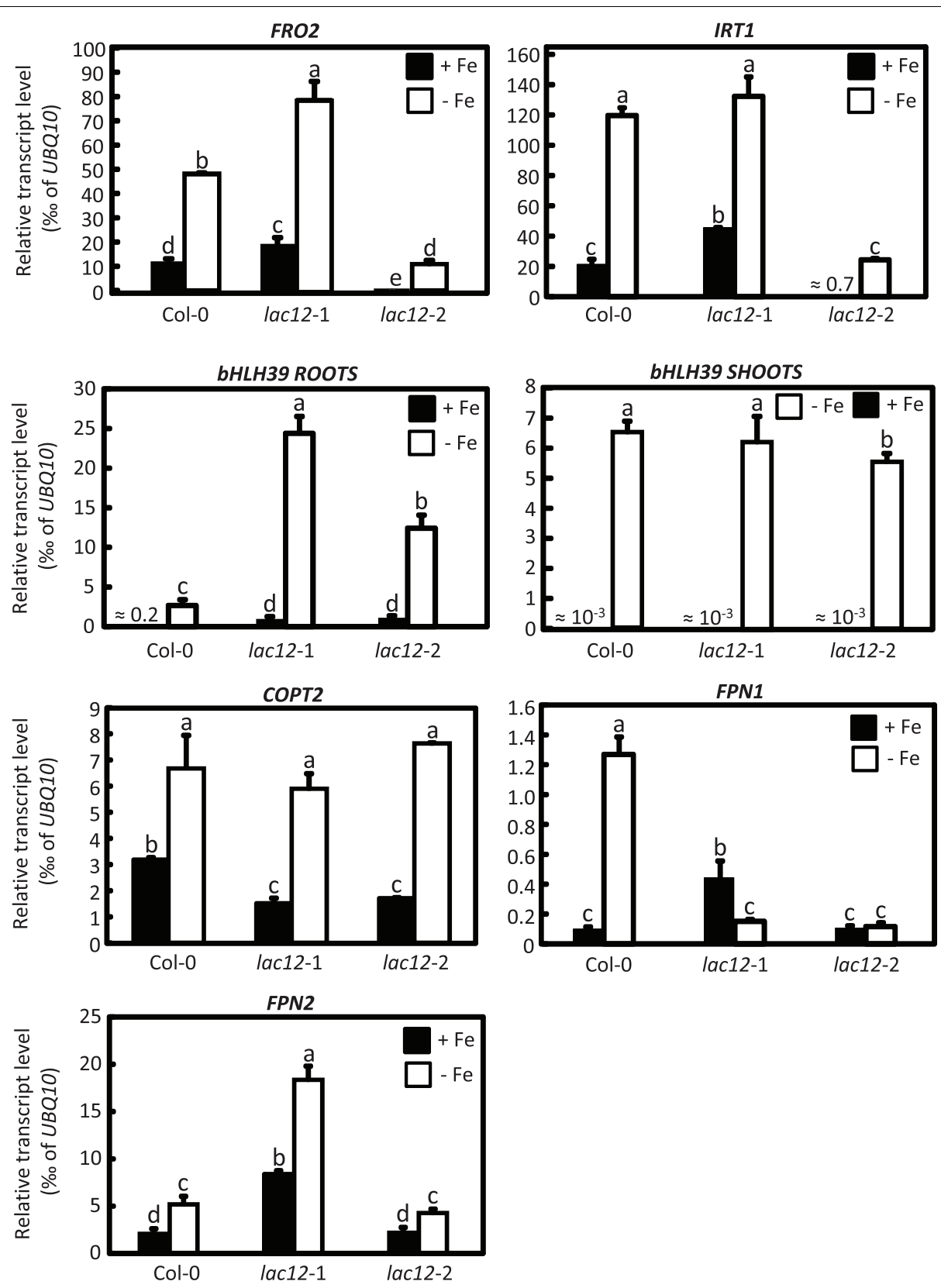

FIGURE 7 | Real time RT-qPCR quantification of several Fe-deficiency responsive transcripts in lac12 mutants. Relative transcript levels of $F R O 2$, IRT1, bHLH39, COPT2, FPN1, and FPN2 in roots and bHLH39 in shoots of WT, lac12-1 and lac12-2 seedlings grown on vertically oriented petri plates as described in Figure $\mathbf{3}$, analyzed by RT-qPCR. Bars show arithmetic means \pm SD of relative transcript levels normalized to UBQ10, and were calculated from $n=4$ technical replicates from one experiment. Different characters denote statistically significant differences $(P<0.05)$ between means based on ANOVA (Tukey's HSD).

known on the importance of ferroxidases in the cellular export of Fe in humans (Bernal et al., 2012). A comparison of the structural superimpositions of LPR1 and LPR2 onto FET3, which allowed to assign the ferroxidase residues in Arabidopsis (Müller et al., 2015) with a BLAST search, alignment and 3D-structure model of LAC12, LAC13 and AO (Table 2 and Supplementary Figures 1, 2A), suggested that LAC3, LAC5, LAC12, LAC13, and AO conserved the essential ferroxidase E185, D283, and D409 residues from yeast as well as the
MCO ferroxidases LPR1 and LPR2 (more details on conserved residue positioning in results) (Müller et al., 2015). Among these proteins, LAC12, LAC13 and AO showed the highest homology to FET3. As a first step toward elucidating the roles of LMCO proteins in $\mathrm{Fe}$ homeostasis of A. thaliana, we focused here primarily on LAC12.

We observed that LAC12 transcript levels were upregulated under Fe deficiency in wild-type plants compared to control conditions (Figure 1) similar to well-known $\mathrm{Fe}$ deficiency 
responsive genes (Figure 7). This result was in agreement with previously published data (Carrió-Seguí et al., 2019b). These authors used plants with modified miR408 levels, a miRNA that mediates the post-transcriptional downregulation of $L A C 3$, LAC12, and LAC13 mRNAs (Abdel-Ghany and Pilon, 2008). They found that under Fe deficiency conditions, all the studied miR408 targets, such as LAC12 and LAC13, were upregulated whereas mir408 was downregulated, in wild-type plants (CarrióSeguí et al., 2019b). Surprisingly, phenoloxidase activity which is classically attributed to laccases, and also ferroxidase activity were decreased under Fe deficiency despite the increase in transcript levels of these two LMCO genes (Carrió-Seguí et al., 2019b). Previous studies showed that mir408 levels change in opposing directions under Fe and $\mathrm{Cu}$ deficiency, respectively (Buhtz et al., 2010; Carrió-Seguí et al., 2019b). On control medium, there was a pronounced increase in LAC12 transcript levels in the irt1 mutant compared to the wild-type (Figure 1). This was consistent with a regulation of $L A C 12$ transcript levels in dependence on plant physiological Fe status, similar to the regulation of a number of other Fe deficiency-responsive genes in roots of the irt1 mutant (Quintana et al., 2021).

The heterologous expression of the LAC12 cDNA restored growth of fet3fet4 yeast cells lacking both the ferroxidase FET3 that is indispensable for high-affinity Fe uptake and FET4mediated low-affinity $\mathrm{Fe}^{2+}$ uptake activity (Figure 2 ). This result suggested that LAC12 may be able to function as MCO with ferroxidase activity that is required to oxidize $\mathrm{Fe}$ (II) to $\mathrm{Fe}$ (III) prior to the uptake of Fe(III) into yeast cells by ScFTR1.

To investigate the role of LAC12 in A. thaliana, two lac12 T-DNA insertion mutants were characterized, in which LAC12 transcript levels were strongly reduced (Supplementary Figure 3). The two lac12 mutants were sensitive to Fe deficiency (Figure 3), with reduced shoot and root biomass as well as strongly decreased leaf chlorophyll concentrations (Figure 3). This suggested that $L A C 12$ function is relevant in the acclimation to Fe deficiency. Under Fe deficiency conditions, lac12 mutant plants also showed higher Fe concentrations in roots compared to wild-type plants (Figure 4A). We could not detect any differences in shoot $\mathrm{Fe}$ concentrations between wild-type and lac12 mutant seedlings (Figure 4B), but total shoot Fe content was lower in lac12 mutants compared to WT seedlings under Fe deficiency conditions (Figure 4C). It was previously suggested that under severe Fe deficiency corresponding to Fe limitation, shoot growth decreases rather than shoot $\mathrm{Fe}$ concentrations (Baxter et al., 2008). This result may alternatively be in agreement with previously published studies in which Fe deficiency, even before detecting any decrease in Fe concentrations, induces the accumulation of $\mathrm{Cu}$ in rosette leaves (Waters et al., 2012; Kastoori Ramamurthy et al., 2018). Perls' stain detected Fe primarily in the stele of the lac12 mutant roots grown under Fe deficiency, but not in wild-type roots (Figure 5). Bernal et al. (2012) reported lowered ferroxidase activities in roots of $\mathrm{Cu}$-deficient spl7 alongside a root-to-shoot Fe translocation defect, and raised the possibility that one or some membrane transport steps in $\mathrm{Fe}$ distribution may depend on MCO-mediated ferroxidase activity. If the decrease in ferroxidase activity in $\mathrm{Cu}$-deficient spl7 was caused solely by impaired LAC12 activity, we would expect Fe to accumulate in the same location in $l a c 12$ and $s p l 7$ roots. Indeed, the localization of Perls' stain was similar in lac12 and spl7 roots, but it was much weaker in lac12 roots (Figure 5). This may indicate that the decrease in ferroxidase activity in $\mathrm{Cu}$-deficient spl7 was not caused solely by impaired LAC12 activity. The site of Fe accumulation in roots of lac12 mutants resembles the site of $L A C 12$ expression according to the public eFP browser, mainly in the vasculature of the root maturation zone adjacent to the xylem (Supplementary Figure 3A) (Brady et al., 2007; Winter et al., 2007). Lower ferroxidase and phenoloxidase activities observed in Fe-deficient lac12 roots (Figure 6) were consistent with the possibility that LAC12 is a MCO with ferroxidase activity.

Root-to-shoot Fe translocation may well require more than the single LAC12 protein, which all depend on SPL7 function under $\mathrm{Cu}$-deficient growth conditions, for example additionally LAC13 or AO, both of them are expressed in the vascular tissues (Supplementary Figure 3A). In the future, generating lac12 lac13 and lac12 ao double mutants will help to clarify their roles. Other candidate proteins that should be examined are LPR1 and LPR2, two MCOs that have a central roles as ferroxidases mediating root phosphate deficiency responses and are expressed, as LAC12 and $L A C 13$, in the vasculature of the root maturation zone adjacent to the xylem (Supplementary Figure 3A) (Müller et al., 2015).

The longitudinal zone of LAC12 expression corresponds to the zone where roots take up Fe from the soil solution. Consequently, the expression pattern of LAC12 in roots is generally in accordance with a speculative role of LAC12 in the movement of $\mathrm{Fe}$ toward or into the xylem. In analogy with MCO proteins of other organisms which have ferroxidase functions, LAC12 could contribute to Fe oxidation either preceding transmembrane $\mathrm{Fe}^{3+}$ uptake into single cells or directly following $\mathrm{Fe}^{2+}$ export from xylem parenchyma cells into xylem vessels, for example. The latter function is more likely, given that no ScFTR1/ScFTR5-like genes are present in the Arabidopsis genome (Figure 8). In this respect, transcript levels of IRON REGULATED 1/FERROPORTIN 1 and 2 (IREG1/FPN1, IREG2/FPN2) were both increased in Fe-deficient WT roots compared to controls (Figure 7), and the expression of FPN1 was downregulated in Fe-deficient roots of lac12 mutants. FPN1 encodes a plasma membranelocalized cellular metal exporter and is expressed in the root stele. Our observation might reflect an interaction of LAC12 and FPN1 protein function at this site, as hypothesized in our working model Figure 8). FPN2 encodes a vacuolar Fe transporter and is expressed in the root epidermis. FPN2 transcript levels are upregulated under Fe-deficient conditions (Morrissey et al., 2009). Recently, FPN3, another member of this Fe exporter family, has been characterized. FPN3 is mainly localized in mitochondria and chloroplasts, and its expression increases under Fe deficiency only in roots (Kim et al., 2021).

A preliminary characterization of the ASCORBATE OXIDASE AO gene, which is also a candidate ferroxidase (see Supplementary Figure 1), suggested that ao mutant seedlings may also be sensitive to Fe deficiency (Supplementary Figure 4). AO is member of the cupredoxin superfamily that has recently been functionally characterized. $A O$, together with three other genes (BBE22, GPX7 and GSTU4), was proposed to act in a complex 


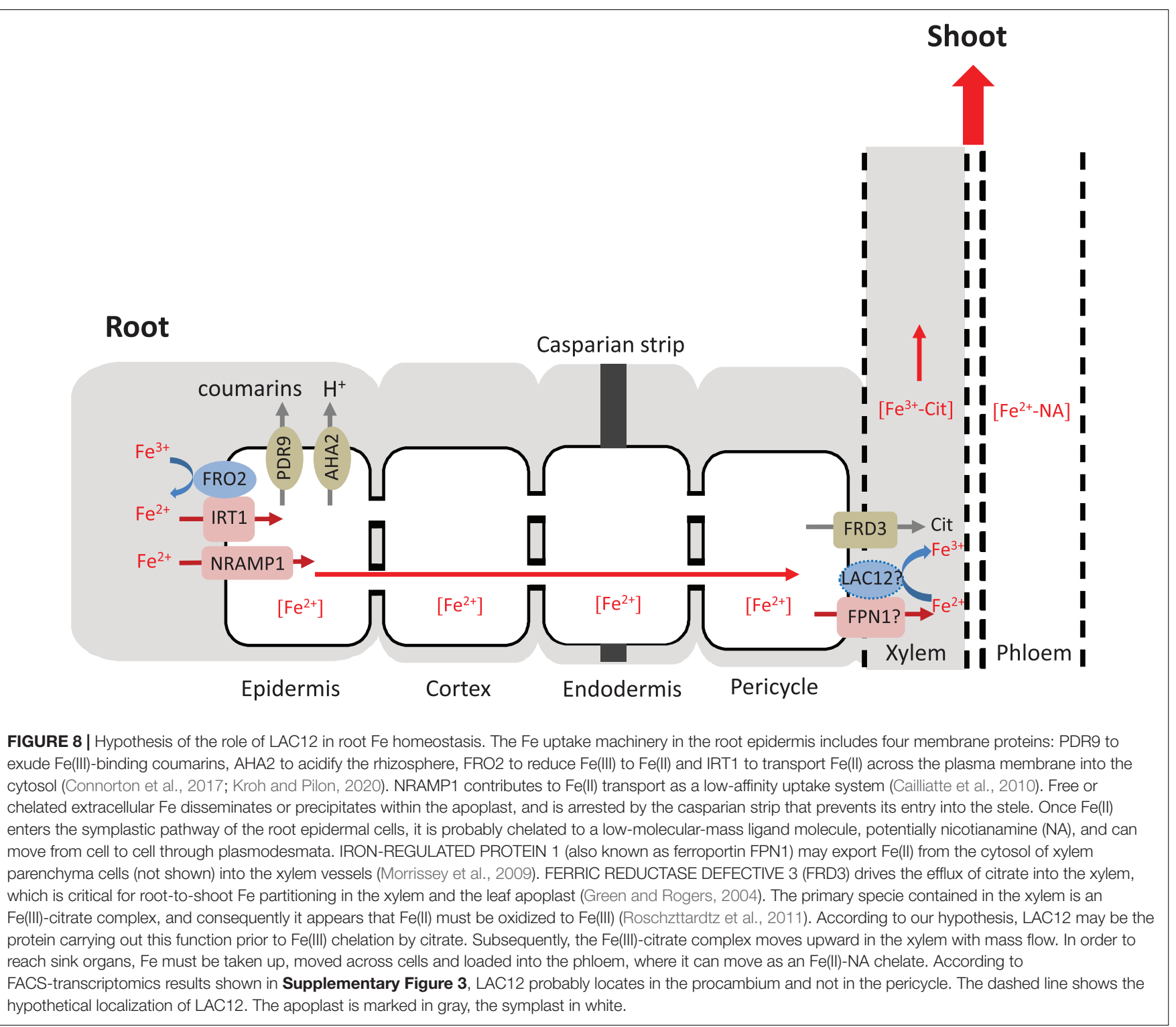

network of ROS-related genes mediating the response of Arabidopsis to the spider mite Tetranychus urticae (Santamaría et al., 2018).

In all genotypes grown under Fe-deficient conditions we observed higher root and shoot $\mathrm{Cu}$ concentrations than upon cultivation under Fe-sufficient conditions (Supplementary Figure 6). Previous studies reported that Fe deficiency, even before detecting any decrease in $\mathrm{Fe}$ concentrations, induces the accumulation of $\mathrm{Cu}$ in rosette leaves (Waters et al., 2012; Kastoori Ramamurthy et al., 2018). Under Fe deficiency, shoot responses require interactions with $\mathrm{Cu}$ accumulation that mediate the replacement of Fe-containing enzymes by $\mathrm{Cu}$-containing enzymes such as SOD (Waters et al., 2012). Root $\mathrm{Cu}$ accumulation under Fe deficiency can be explained by $\mathrm{Cu}$ uptake via enhanced expression of genes encoding ZRT/IRT-LIKE PROTEIN2 (ZIP2) and COPT2 membrane transporters, which can mediate the cellular uptake of $\mathrm{Cu}(\mathrm{II})$ or $\mathrm{Cu}(\mathrm{I})$, respectively (Colangelo and Guerinot, 2004; Perea-García et al., 2013).

After a careful analysis of the different studies regarding the cross-talk between $\mathrm{Fe}$ and $\mathrm{Cu}$ homeostasis in plants, we must admit that the relationship among them is very complex. On the one hand, our previous publication showed that $\mathrm{Cu}$ deficiency drives Fe accumulation in the root of wild-type and spl7 mutant (Bernal et al., 2012). Another laboratory reported that $\mathrm{Cu}$ deficient spl7 mutant leaves accumulate more Fe, and that lowering Fe supply rescues the growth phenotype of spl7 mutants (Kastoori Ramamurthy et al., 2018). Additionally, ysl3 mutants of $B$. distachyon accumulated more $\mathrm{Fe}$ in roots and leaves under $\mathrm{Cu}$ deficient conditions (Sheng et al., 2021). One main difference that could explain the different results obtained by different research groups is the Fe chelator used in the Hoagland's growth medium: Fe(III)-EDDHA (Kastoori Ramamurthy et al., 2018; Sheng et al., 2021) versus FeHBED (Bernal et al., 2012). Our laboratory 
uses HBED ( $N, N^{\prime}$-di(2-hydroxybenzyl)ethylenediamine$N, N^{\prime}$-diacetic acid) for $\mathrm{Fe}$ chelation in plant growth media. This chelator shows only a limited affinity for $\mathrm{Cu}$ and remains chelated to $\mathrm{Fe}$ (III) over a wide $\mathrm{pH}$ range. However, EDDHA has a higher affinity for $\mathrm{Cu}$ than for $\mathrm{Fe}(\mathrm{III})$ and when added to the growth media can therefore restrict the bioavailability of $\mathrm{Cu}$, among other micronutrients (i.e., $\mathrm{Zn}$ ), and result in an effective deficiency of these metals. Lowering Fe(III)EDDHA means lowering also EDDHA and thus decreasing $\mathrm{Cu}$ chelation. Taking this in consideration, the rescue of the spl7 phenotype by low Fe supply (Kastoori Ramamurthy et al., 2018) may be correlated with the decrease of the concentration of a $\mathrm{Cu}$ chelator and not with lowering Fe concentration itself. In fact, we believe that the strong phenotype of the spl7 mutant grown under $0.1 \mu \mathrm{M} \mathrm{Cu}$ and $25 \mu \mathrm{M}$ Fe (Kastoori Ramamurthy et al., 2018) is caused by both, the low $\mathrm{Cu}$ concentrations and the effect that $\mathrm{Fe}(\mathrm{III})-\mathrm{EDDHA}$ has on the bioavailability of $\mathrm{Cu}$.

\section{CONCLUSION}

Our initial characterization of LACCASE12 (LAC12), a member of the laccase multicopper oxidase (LMCO) protein family, provides evidence for a role in the performance of Arabidopsis on Fe-deficient substrates and initial support for a ferroxidase function of the encoded protein. Alterations in root $\mathrm{Fe}$ concentrations and total shoot $\mathrm{Fe}$ of lac12 mutants are consistent with a possible function of LAC12 in root-toshoot Fe partitioning and a possible role in enabling the cellular export of Fe. More work will clearly be needed to understand the role of MCO proteins LAC12 and the AO in $\mathrm{Fe}$ homeostasis. Unraveling the interactions between $\mathrm{Cu}$ and $\mathrm{Fe}$ homeostasis may lead to the development of novel strategies for combating nutritional $\mathrm{Fe}$ deficiencies in crops and for advancing biofortification approaches.

\section{DATA AVAILABILITY STATEMENT}

The original contributions presented in the study are included in the article/Supplementary Material, further inquiries can be directed to the corresponding authors.

\section{REFERENCES}

Abdel-Ghany, S. E., and Pilon, M. (2008). MicroRNA-mediated systemic downregulation of copper protein expression in response to low copper availability in Arabidopsis. J. Biol. Chem. 283, 15932-15945. doi: 10.1074/jbc.m801406200

Altschul, S. F., and Lipman, D. J. (1990). Protein database searches for multiple alignments. Proc. Natl. Acad. Sci. U. S. A. 87, 5509-5513. doi: 10.1073/pnas.87. 14.5509

Andrés-Bordería, A., Andrés, F., Garcia-Molina, A., Perea-García, A., Domingo, C., Puig, S., et al. (2017). Copper and ectopic expression of the Arabidopsis transport protein COPT1 alter iron homeostasis in rice (Oryza sativa L.). Plant. Mol. Biol. 95, 17-32. doi: 10.1007/s11103-017-0622-8

Askwith, C., Eide, D., Van Ho, A., Bernard, P. S., Li, L., Davis-Kaplan, S., et al. (1994). The FET3 gene of S. cerevisiae encodes a multicopper oxidase required for ferrous iron uptake. Cell 76, 403-410. doi: 10.1016/0092-8674(94)9 0346-8

\section{AUTHOR CONTRIBUTIONS}

$\mathrm{MB}$ and $\mathrm{UK}$ conceived the project, designed research, and analyzed data. $\mathrm{MB}$ performed experiments and wrote the manuscript. UK commented on and edited the manuscript. Both authors contributed to the article and approved the submitted version.

\section{FUNDING}

This work was supported by the Deutsche Forschungsgemeinschaft grants Kr1967/3-3 and 15-1, European Union FOOD-CT2006-016253 and ERC-AdG LEAP EXTREME (788380) to UK, Ruhr University Bochum (UK), Araid-Ibercaja-young scientist grant 2010 (MB), as well as Juan de la Cierva (MICINN) and JAE-DOC (CSIC) (MB).

\section{ACKNOWLEDGMENTS}

We thank David Eide (University of Wisconsin-Madison, United States) for providing the fet 3 fet 4 and corresponding wildtype yeast strains and Dario Leister (LM University Munich, Germany) for Arabidopsis pam42 seeds. We thank Petra Düchting for multi-element analysis, and Andreas Aufermann and Iris Sandorf for supporting plant cultivation and all lab members for their useful comments (RUB). We also thank María A. Luján and Patricia Lorente for technical assistance, and Rafael Picorel for providing infrastructure (EEAD, CSIC, Spain). We acknowledge support by the Open Access Publication Funds of the Ruhr-Universität Bochum.

\section{SUPPLEMENTARY MATERIAL}

The Supplementary Material for this article can be found online at: https://www.frontiersin.org/articles/10.3389/fpls.2021. 688318/full\#supplementary-material

Baxter, I. R., Vitek, O., Lahner, B., Muthukumar, B., Borghi, M., Morrissey, J., et al. (2008). The leaf ionome as a multivariable system to detect a plant's physiological status. Proc. Natl. Acad. Sci. U. S. A. 105, 12081-12086. doi: 10.1073/pnas.0804175105

Beauclair, L., Yu, A., and Bouché, N. (2010). microRNA-directed cleavage and translational repression of the copper chaperone for superoxide dismutase mRNA in Arabidopsis. Plant J. 62, 454-462. doi: 10.1111/j.1365-313X.2010. 04162.x

Bernal, M., Casero, D., Singh, V., Wilson, G. T., Grande, A., Yang, H., et al. (2012). SPL7-regulated copper acquisition genes FRO4 /FRO5 (FRO4/FRO5) and the copper dependence of iron homeostasis in Arabidopsis. Plant Cell 24, 738-761. doi: 10.1105/tpc.111.090431

Bonaccorsi di Patti, M. C., Felice, M. R., De Domenico, I., Lania, A., Alaleona, F., et al. (2005). Specific aspartate residues in FET3 control high-affinity iron transport in Saccharomyces cerevisiae. Yeast 22, 677-687. doi: 10.1002/yea. 1237 
Brady, S. M., Orlando, D. A., Lee, J. Y., Wang, J. Y., Koch, J., Dinneny, J. R., et al. (2007). A high-resolution root spatiotemporal map reveals dominant expression patterns. Science 318, 801-806. doi: 10.1126/science.1146265

Brumbarova, T., and Ivanov, R. (2014). Perls staining for histochemical detection of iron in plant samples. Bio-Protocol 4:e1245. doi: 10.21769/BioProtoc. 1245

Buckhout, T. J., Yang, T. J. W., and Schmidt, W. (2009). Early iron-deficiencyinduced transcriptional changes in Arabidopsis roots as revealed by microarray analyses. BMC Genomics 10:147. doi: 10.1186/1471-2164-10-147

Buhtz, A., Pieritz, J., Springer, F., and Kehr, J. (2010). Phloem small RNAs, nutrient stress responses, and systemic mobility. BMC Plant Biol. 10:64. doi: 10.1186/ 1471-2229-10-64

Cailliatte, R., Schikora, A., Briat, J. F., Mari, S., and Curie, C. (2010). Highaffinity manganese uptake by the metal transporter NRAMP1 is essential for Arabidopsis growth in low manganese conditions. Plant Cell 22, 904-917. doi: 10.1105/tpc.109.073023

Carrió-Seguí, À, Romero, P., Curie, C., Mari, S., and Peñarrubia, L. (2019a). Copper transporter COPT5 participates in the crosstalk between vacuolar copper and iron pools mobilisation. Sci. Rep. 9:4648. doi: 10.1038/s41598-018-3 8005-4

Carrió-Seguí, À, Ruiz-Rivero, O., Villamayor-Belinchón, L., Puig, S., Perea-García, A., and Peñarrubia, L. (2019b). The altered expression of microRNA408 influences the Arabidopsis response to iron deficiency. Front. Plant Sci. 10:324. doi: 10.3389/fpls.2019.00324

Chen, H., Attieh, Z., Syed, B., Kuo, Y.-M., Currie, V., Fuqua, B., et al. (2010). Identification of zyklopen, a new member of the vertebrate multicopper ferroxidase family, and characterization in rodents and human cells. J. Nutr. 140, 1728-1735. doi: 10.3945/jn.109.117531

Colangelo, E. P., and Guerinot, M. L. (2004). The essential basic helix-loophelix protein FIT1 is required for the iron deficiency response. Plant Cell 16, 3400-3412. doi: 10.1105/tpc.104.024315

Collins, J. F., Prohaska, J. R., and Knutson, M. D. (2010). Metabolic crossroads of iron and copper. Nutr. Rev. 68, 133-147.

Connolly, E. L., Fett, J. P., and Guerinot, M. L. (2002). Expression of the IRT1 metal transporter is controlled by metals at the levels of transcript and protein accumulation. Plant Cell 14, 1347-1357. doi: 10.1105/tpc.001263

Connorton, J. M., Balk, J., and Rodríguez-Celma, J. (2017). Iron homeostasis in plants - a brief overview. Metallomics 9, 813-823. doi: 10.1039/c7mt00136c

Desbrosses-Fonrouge, A. G., Voigt, K., Schröder, A., Arrivault, S., Thomine, S. and Krämer, U. (2005). Arabidopsis thaliana MTP1 is a Zn transporter in the vacuolar membrane which mediates $\mathrm{Zn}$ detoxification and drives leaf $\mathrm{Zn}$ accumulation. FEBS Lett. 579, 4165-4174. doi: 10.1016/j.febslet.2005.06.046

Dix, D. R., Bridgham, J. T., Broderius, M. A., Byersdorfer, C. A., and Eide, D. J. (1994). The FET4 gene encodes the low affinity Fe(II) transport protein of Saccharomyces cerevisiae. J. Biol. Chem. 269, 26092-26099. doi: 10.1016/s00219258(18)47163-3

Dohmen, R. J., Strasser, A. W., Höner, C. B., and Hollenberg, C. P. (1991). An efficient transformation procedure enabling long-term storage of competent cells of various yeast genera. Yeast 7, 691-692. doi: 10.1002/yea.32 0070704

Dusek, P., Roos, P. M., Litwin, T., Schneider, S. A., Flaten, T. P., and Aaseth, J. (2015). The neurotoxicity of iron, copper and manganese in Parkinson's and Wilson's diseases. J. Trace Elem. Med. Biol. 31, 193-203. doi: 10.1016/j.jtemb. 2014.05.007

Edwards, K., Johnstone, C., and Thompson, C. (1991). A simple and rapid method for the preparation of plant genomic DNA for PCR analysis. Nucleic. Acids Res. 19:1349. doi: 10.1093/nar/19.6.1349

Eide, D., Broderius, M., Fett, J., and Guerinot, M. L. (1996). A novel iron-regulated metal transporter from plants identified by functional expression in yeast. Proc. Natl. Acad. Sci. U. S. A. 93, 5624-5628. doi: 10.1073/pnas.93.11.5624

Garcia-Molina, A., Lehmann, M., Schneider, K., Klingl, A., and Leister, D. (2021). Inactivation of cytosolic FUMARASE2 enhances growth and photosynthesis under simultaneous copper and iron deprivation in Arabidopsis. Plant J. 106, 766-784. doi: 10.1111/tpj.15199

Garcia-Molina, A., Marino, G., Lehmann, M., and Leister, D. (2020). Systems biology of responses to simultaneous copper and iron deficiency in Arabidopsis. Plant J. 103, 2119-2138. doi: 10.1111/tpj.14887

Green, L. S., and Rogers, E. E. (2004). FRD3 controls iron localization in Arabidopsis. Plant Physiol. 136, 2523-2531. doi: 10.1104/pp.104.045633
Gulec, S., and Collins, J. F. (2014). Molecular mediators governing iron-copper interactions. Annu. Rev. Nutr. 34, 95-116. doi: 10.1146/annurev-nutr-071812161215

Harris, Z. L., Takahashi, Y., Miyajima, H., Serizawa, M., MacGillivray, R. T., and Gitlin, J. D. (1995). Aceruloplasminemia: molecular characterization of this disorder of iron metabolism. Proc. Natl. Acad. Sci. U. S. A. 92, 2539-2543. doi: 10.1073/pnas.92.7.2539

Haydon, M. J., Kawachi, M., Wirtz, M., Hillmer, S., Hell, R., and Krämer, U. (2012). Vacuolar nicotianamine has critical and distinct roles under iron deficiency and for zinc sequestration in Arabidopsis. Plant Cell 24, 724-737. doi: 10.1105/tpc. 111.095042

Hellman, N. E., and Gitlin, J. D. (2002). Ceruloplasmin metabolism and function. Annu. Rev. Nutr. 22, 439-458. doi: 10.1146/annurev.nutr.22.012502.11 4457

Henriques, R., Jásik, J., Klein, M., Martinoia, E., Feller, U., Schell, J., et al. (2002). Knock-out of Arabidopsis metal transporter gene IRT1 results in iron deficiency accompanied by cell differentiation defects. Plant Mol. Biol. 50, 587-597. doi: 10.1023/a:1019942200164

Kastoori Ramamurthy, R., Xiang, Q., Hsieh, E.-J., Liu, K., Zhang, C., and Waters, B. M. (2018). New aspects of iron-copper crosstalk uncovered by transcriptomic characterization of Col-0 and the copper uptake mutant $s p l 7$ in Arabidopsis thaliana. Metallomics 10, 1824-1840. doi: 10.1039/c8mt00287h

Katoh, K., Misawa, K., Kuma, K., and Miyata, T. (2002). MAFFT: a novel method for rapid multiple sequence alignment based on fast Fourier transform. Nucleic Acids Res. 30, 3059-3066. doi: 10.1093/nar/gkf436

Kelley, L. A., and Sternberg, M. J. (2009). Protein structure prediction on the Web: a case study using the Phyre server. Nat. Protocols 4, 363-371.

Kim, L. J., Tsuyuki, K. M., Hu, F., Park, E. Y., Zhang, J., Iraheta, J. G., et al. (2021). Ferroportin 3 is a dual-targeted mitochondrial/chloroplast iron exporter necessary for iron homeostasis in Arabidopsis. Plant J. 107, 215-236. doi: 10. 1111/tpj.15286

Kobayashi, T., and Nishizawa, N. K. (2012). Iron uptake, translocation, and regulation in higher plants. Annu. Rev. Plant Bio. 63, 131-152. doi: 10.1146/ annurev-arplant-042811-105522

Kroh, G. E., and Pilon, M. (2020). Regulation of iron homeostasis and use in chloroplasts. Int. J. Mol. Sci. 21:3395. doi: 10.3390/ijms21093395

Kropat, J., Tottey, S., Birkenbihl, R. P., Depege, N., Huijser, P., and Merchant, S. (2005). A regulator of nutritional copper signaling in Chlamydomonas is an SBP domain protein that recognizes the GTAC core of copper response element. Proc. Natl. Acad. Sci. U. S. A. 102, 18730-18735. doi: 10.1073/pnas.0507693102

La Fontaine, S., Quinn, J. M., Nakamoto, S. S., Page, M. D., Gohre, V., Moseley, J. L., et al. (2002). Copper-dependent iron assimilation pathway in the model photosynthetic eukaryote Chlamydomonas reinhardtii. Eukaryot. Cell 1, 736757. doi: $10.1128 /$ ec.1.5.736-757.2002

Marschner, H. (1995). Mineral Nutrition of Higher Plants. Boston: Academic Press. Masuda, H., Aung, M. S., and Nishizawa, N. K. (2013). Iron biofortification of rice using different transgenic approaches. Rice 6, 40. doi: 10.1186/1939-84 33-6-40

McCaig, B. C., Meagher, R. B., and Dean, J. F. (2005). Gene structure and molecular analysis of the laccase-like multicopper oxidase (LMCO) gene family in Arabidopsis thaliana. Planta 221, 619-636. doi: 10.1007/s00425-0041472-6

Morrissey, J., Baxter, I. R., Lee, J., Li, L., Lahner, B., Grotz, N., et al. (2009). The ferroportin metal efflux proteins function in iron and cobalt homeostasis in Arabidopsis. Plant Cell 21, 3326-3338. doi: 10.1105/tpc.109.069401

Muckenthaler, M. U., Galy, B., and Hentze, M. W. (2008). Systemic iron homeostasis and the iron-responsive element/iron-regulatory protein (IRE/IRP) regulatory network. Annu. Rev. Nutr. 28, 197-213. doi: 10.1146/annurev.nutr.28.061807.155521

Müller, J., Toev, T., Heisters, M., Teller, J., Moore, Katie, L., et al. (2015). Irondependent callose deposition adjusts root meristem maintenance to phosphate availability. Dev. Cell 33, 216-230. doi: 10.1016/j.devcel.2015.02.007

Nouet, C., Motte, P., and Hanikenne, M. (2011). Chloroplastic and mitochondrial metal homeostasis. Trends Plant Sci. 16, 395-404. doi: 10.1016/j.tplants.2011. 03.005

Nriagu, J. O., and Pacyna, J. M. (1988). Quantitative assessment of worldwide contamination of air, water and soils by trace metals. Nature 333, 134-139. doi: $10.1038 / 333134 \mathrm{a} 0$ 
Pan, J., Huang, D., Guo, Z., Kuang, Z., Zhang, H., Xie, X., et al. (2018). Overexpression of microRNA408 enhances photosynthesis, growth, and seed yield in diverse plants. J. Integr. Plant Biol. 60, 323-340. doi: 10.1111/jipb.12634

Perea-García, A., Andrés-Bordería, A., Vera-Sirera, F., Pérez-Amador, M. A., Puig, S., and Peñarrubia, L. (2020). Deregulated high affinity copper transport alters iron homeostasis in Arabidopsis. Front. Plant Sci. 11:1106. doi: 10.3389/fpls. 2020.01106

Perea-García, A., Garcia-Molina, A., Andrés-Colás, N., Vera-Sirera, F., PérezAmador, M. A., Puig, S., et al. (2013). Arabidopsis copper transport protein COPT2 participates in the cross talk between iron deficiency responses and low-phosphate signaling. Plant Physiol. 162, 180-194. doi: 10.1104/pp.112. 212407

Pilon, M. (2017). The copper microRNAs. New Phytol. 213, 1030-1035. doi: 10. $1111 /$ nph.14244

Porra, R. J., Thompson, W. A., and Kriedemann, P. E. (1989). Determination of accurate extinction coefficients and simultaneous equations for assaying chlorophylls $\mathrm{a}$ and $\mathrm{b}$ extracted with four different solvents: verification of the concentration of chlorophyll standards by atomic absorption spectroscopy. Biochim. Biophys. Acta Bioenerg. 975, 384-394. doi: 10.1016/s0005-2728(89) 80347-0

Printz, B., Lutts, S., Hausman, J.-F., and Sergeant, K. (2016). Copper trafficking in plants and its implication on cell wall dynamics. Front. Plant Sci. 7:601. doi: 10.3389/fpls.2016.00601

Puig, S., Andrés-Colás, N., García-Molina, A., and Peñarrubia, L. (2007). Copper and iron homeostasis in Arabidopsis: responses to metal deficiencies, interactions and biotechnological applications. Plant Cell Environ. 30, 271-290. doi: $10.1111 /$ j.1365-3040.2007.01642.x

Quinn, J. M., and Merchant, S. S. (1995). Two copper-responsive elements associated with the Chlamydomonas Cyc6 gene function as targets for transcriptional activators. Plant Cell 7, 623-628. doi: 10.2307/3870120

Quintana, J., Bernal, M. I., Scholle, M., Holländer-Czytko, H., Nga, N., Piotrowski, M., et al. (2021). Root-to-shoot iron partitioning in Arabidopsis requires IRONREGULATED TRANSPORTER1 (IRT1). bioRxiv [Preprint]. doi: 10.1101/2021. 02.08.430285

Quintanar, L., Stoj, C., Taylor, A. B., Hart, P. J., Kosman, D. J., and Solomon, E. I. (2007). Shall we dance? How a multicopper oxidase chooses its electron transfer partner. Acc. Chem. Res. 40, 445-452. doi: 10.1021/ar600051a

Ravet, K., and Pilon, M. (2013). Copper and iron homeostasis in plants: the challenges of oxidative stress. Antioxid. Redox Signal. 19, 919-932. doi: 10.1089/ ars.2012.5084

Reiss, R., Ihssen, J., Richter, M., Eichhorn, E., Schilling, B., and Thöny-Meyer, L. (2013). Laccase versus laccase-like multi-copper oxidase: a comparative study of similar enzymes with diverse substrate spectra. PLoS One 8:e65633. doi: 10.1371/journal.pone.0065633

Rogers, E. E., Eide, D. J., and Guerinot, M. L. (2000). Altered selectivity in an Arabidopsis metal transporter. Proc. Natl. Acad. Sci. U. S. A. 97, 12356-12360. doi: $10.1073 /$ pnas.210214197

Rojas-Murcia, N., Hématy, K., Lee, Y., Emonet, A., Ursache, R., Fujita, S., et al. (2020). High-order mutants reveal an essential requirement for peroxidases but not laccases in Casparian strip lignification. Proc. Natl. Acad. Sci. U. S. A. 117, 29166-29177. doi: 10.1073/pnas.2012728117

Roschzttardtz, H., Conéjéro, G., Curie, C., and Mari, S. (2009). Identification of the endodermal vacuole as the iron storage compartment in the Arabidopsis embryo. Plant Physiol. 151, 1329-1338. doi: 10.1104/pp.109.144444

Roschzttardtz, H., Séguéla-Arnaud, M., Briat, J.-F., Vert, G., and Curie, C. (2011). The FRD3 citrate effluxer promotes iron nutrition between symplastically disconnected tissues throughout Arabidopsis development. Plant Cell 23, 27252737. doi: $10.1105 /$ tpc. 111.088088

Ruijter, J. M., Ramakers, C., Hoogaars, W. M., Karlen, Y., Bakker, O., van den Hoff, M. J., et al. (2009). Amplification efficiency: linking baseline and bias in the analysis of quantitative PCR data. Nucleic Acids Res. 37:e45.

Sancenón, V., Puig, S., Mira, H., Thiele, D. J., and Peñarrubia, L. (2003). Identification of a copper transporter family in Arabidopsis thaliana. Plant Mol. Biol. 51, 577-587.

Santamaría, M. E., Arnaiz, A., Velasco-Arroyo, B., Grbic, V., Diaz, I., and Martinez, M. (2018). Arabidopsis response to the spider mite Tetranychus urticae depends on the regulation of reactive oxygen species homeostasis. Sci. Rep. 8:9432. doi: 10.1038/s41598-018-27904-1
Schwacke, R., Schneider, A., van der Graaff, E., Fischer, K., Catoni, E., Desimone, M., et al. (2003). ARAMEMNON, a novel database for Arabidopsis integral membrane proteins. Plant Physiol. 131, 16-26. doi: 10.1104/pp. 011577

Shahzad, Z., Rouached, H., and Rakha, A. (2014). Combating mineral malnutrition through iron and zinc biofortification of cereals. Compr. Rev. Food Sci. Food Saf. 13, 329-346. doi: 10.1111/1541-4337.12063

Sheng, H., Jiang, Y., Rahmati, M., Chia, J.-C., Dokuchayeva, T., Kavulych, Y., et al. (2021). YSL3-mediated copper distribution is required for fertility, seed size and protein accumulation in Brachypodium. Plant Physiol. 186, 655-676. doi: 10.1093/plphys/kiab054

Sinclair, S. A., Larue, C., Bonk, L., Khan, A., Castillo-Michel, H., Stein, R. J., et al. (2017). Etiolated seedling development requires repression of photomorphogenesis by a small cell-wall-derived dark signal. Curr. Biol. 27, 3403-3418.e7. doi: 10.1016/j.cub.2017.09.063

Song, Z., Zhang, L., Wang, Y., Li, H., Li, S., Zhao, H., et al. (2018). Constitutive expression of miR408 improves biomass and seed yield in Arabidopsis. Front. Plant Sci. 8:2114. doi: 10.3389/fpls.2017.02114

Sunkar, R., and Zhu, J.-K. (2004). Novel and stress-regulated microRNAs and other small RNAs from Arabidopsis. Plant Cell 16, 2001-2019. doi: 10.1105/tpc.104. 022830

Svistoonoff, S., Creff, A., Reymond, M., Sigoillot-Claude, C., Ricaud, L., Blanchet, A., et al. (2007). Root tip contact with low-phosphate media reprograms plant root architecture. Nat. Genet. 39, 792-796. doi: 10.1038/ng2041

Taylor, A. B., Stoj, C. S., Ziegler, L., Kosman, D. J., and Hart, P. J. (2005). The copper-iron connection in biology: structure of the metallo-oxidase Fet3p. Proc. Natl. Acad. Sci. U. S. A. 102, 15459-15464. doi: 10.1073/pnas.0506227102

Tewari, R. K., Kumar, P., and Sharma, P. N. (2006). Antioxidant responses to enhanced generation of superoxide anion radical and hydrogen peroxide in the copper-stressed mulberry plants. Planta 223, 1145-1153. doi: 10.1007/s00425005-0160-5

Ticconi, C. A., Lucero, R. D., Sakhonwasee, S., Adamson, A. W., Creff, A., Nussaume, L., et al. (2009). ER-resident proteins PDR2 and LPR1 mediate the developmental response of root meristems to phosphate availability. Proc. Natl. Acad. Sci. U. S. A. 106, 14174-14179. doi: 10.1073/pnas.0901778106

Turlapati, P. V., Kim, K. W., Davin, L. B., and Lewis, N. G. (2011). The laccase multigene family in Arabidopsis thaliana: towards addressing the mystery of their gene function(s). Planta 233, 439-470. doi: 10.1007/s00425-010-1298-3

Varotto, C., Maiwald, D., Pesaresi, P., Jahns, P., Salamini, F., and Leister, D. (2002). The metal ion transporter IRT1 is necessary for iron homeostasis and efficient photosynthesis in Arabidopsis thaliana. Plant J. 31, 589-599. doi: 10.1046/j. 1365-313x.2002.01381.x

Vert, G., Grotz, N., Dédaldéchamp, F., Gaymard, F., Guerinot, M. L., Briat, J. F., et al. (2002). IRT1, an Arabidopsis transporter essential for iron uptake from the soil and for plant growth. Plant Cell 14, 1223-1233. doi: 10.1105/tpc.001388

Ward, J. T., Lahner, B., Yakubova, E., Salt, D. E., and Raghothama, K. G. (2008). The effect of iron on the primary root elongation of Arabidopsis during phosphate deficiency. Plant Physiol. 147, 1181-1191. doi: 10.1104/pp.108.118562

Warren, C. R. (2008). Rapid measurement of chlorophylls with a microplate reader. J. Plant Nutr. 31, 1321-1332. doi: 10.1080/01904160802135092

Wass, M. N., Kelley, L. A., and Sternberg, M. J. (2010). 3DLigandSite: predicting ligand-binding sites using similar structures. Nucleic Acids Res. 38, W469W473. doi: 10.1093/nar/gkq406

Waters, B. M., McInturf, S. A., and Stein, R. J. (2012). Rosette iron deficiency transcript and microRNA profiling reveals links between copper and iron homeostasis in Arabidopsis thaliana. J. Exp. Bot. 63, 5903-5918. doi: 10.1093/ jxb/ers239

Winter, D., Vinegar, B., Nahal, H., Ammar, R., Wilson, G. V., and Provart, N. J. (2007). An "electronic fluorescent pictograph" browser for exploring and analyzing large-scale biological data sets. PLoS One 2:e718. doi: 10.1371/journal. pone. 0000718

Yamasaki, H., Abdel-Ghany, S. E., Cohu, C. M., Kobayashi, Y., Shikanai, T., and Pilon, M. (2007). Regulation of copper homeostasis by micro-RNA in Arabidopsis. J. Biol. Chem. 282, 16369-16378. doi: 10.1074/jbc.m700138200

Yamasaki, H., Hayashi, M., Fukazawa, M., Kobayashi, Y., and Shikanai, T. (2009). SQUAMOSA Promoter Binding Protein-Like7 is a central regulator for copper homeostasis in Arabidopsis. Plant Cell 21, 347-361. doi: 10.1105/tpc.108. 060137 
Zhang, H., and Li, L. (2013). SQUAMOSA promoter binding protein-like7 regulated microRNA408 is required for vegetative development in Arabidopsis. Plant J. 74, 98-109. doi: 10.1111/tpj.12107

Zhang, H., Zhao, X., Li, J., Cai, H., Deng, X. W., and Li, L. (2014). MicroRNA408 is critical for the HY5-SPL7 gene network that mediates the coordinated response to light and copper. Plant Cell 26, 4933-4953. doi: 10.1105/tpc.114.1 27340

Zhang, J. P., Yu, Y., Feng, Y. Z., Zhou, Y. F., Zhang, F., Yang, Y. W., et al. (2017). MiR408 regulates grain yield and photosynthesis via a phytocyanin protein. Plant Physiol. 175, 1175-1185. doi: 10.1104/pp.17.01169

Zhao, F.-J., and McGrath, S. P. (2009). Biofortification and phytoremediation. Curr. Opin. Plant Biol. 12, 373-380. doi: 10.1016/j.pbi.2009.04.005

Zhuang, Y., Zuo, D., Tao, Y., Cai, H., and Li, L. (2020). Laccase3-based extracellular domain provides possible positional information for directing Casparian strip formation in Arabidopsis. Proc. Natl. Acad. Sci. U. S. A. 117, 15400-15402. doi: 10.1073/pnas.2005429117
Conflict of Interest: The authors declare that the research was conducted in the absence of any commercial or financial relationships that could be construed as a potential conflict of interest.

Publisher's Note: All claims expressed in this article are solely those of the authors and do not necessarily represent those of their affiliated organizations, or those of the publisher, the editors and the reviewers. Any product that may be evaluated in this article, or claim that may be made by its manufacturer, is not guaranteed or endorsed by the publisher.

Copyright (C) 2021 Bernal and Krämer. This is an open-access article distributed under the terms of the Creative Commons Attribution License (CC BY). The use, distribution or reproduction in other forums is permitted, provided the original author(s) and the copyright owner(s) are credited and that the original publication in this journal is cited, in accordance with accepted academic practice. No use, distribution or reproduction is permitted which does not comply with these terms. 